\title{
UNIVERSAL CONVEXITY FOR QUASIHYPERBOLIC TYPE METRICS
}

\author{
DAVID A. HERRON \\ Dedicated to Taft Professor David Minda on the occasion of his retirement.
}

\begin{abstract}
We characterize the open sets in the sphere that are geodesically convex in any containing domain with respect to various conformal metrics.
\end{abstract}

\section{INTRODUCTION}

Determining the location of geodesics is an important problem in metric geometry. For example, if we conformally deform a metric space, we can ask whether balls in the original geometry are geodesically convex in the new geometry. To be precise, let $\Omega$ be a domain in the sphere $\hat{\mathrm{R}}^{n}:=\mathrm{R}^{n} \cup\{\infty\}(n \geq 2)$, suppose $\rho d s$ is a conformal metric on $\Omega$, and consider the conformal deformation $\Omega_{\rho}:=\left(\Omega, d_{\rho}\right)$ of $\Omega$ where $d_{\rho}$ is the length distance given via

$$
d_{\rho}(x, y):=\inf _{\gamma} \ell_{\rho}(\gamma) \text { with } \ell_{\rho}(\gamma):=\int_{\gamma} \rho d s
$$

here the infimum is taken over all rectifiable paths $\gamma$ in $\Omega$ that join the points $x, y$. Any such path $\gamma$ that has $\rho$-length $\ell_{\rho}(\gamma)=d_{\rho}(x, y)$ is a $\rho$-geodesic. (See Section 2 for more definitions and notation).

We ask: When is a ball in $\Omega$ convex in $\Omega_{\rho}$ ? Here $A \subset \Omega$ is convex in $\Omega_{\rho}$, or, $\rho$-convex, if it is geodesically convex in $\Omega_{\rho}$. This means that for all points $x, y$ in $A$, every $\rho$-geodesic $[x, y]_{\rho}$ lies in $A$. (Recall that an open ball in $\hat{\mathrm{R}}^{n}$-a.k.a., a Möbius ball - is an open Euclidean ball or an open half-space (both in $\mathrm{R}^{n}$ ) or the complement of a closed Euclidean ball.)

For example, in 1956 Vilhelm Jørgensen proved that if $D$ is a disk (in $\hat{C}$ ) and $D \subset \Omega$, then $D$ is $h$-convex in $\Omega$ where $h=h_{\Omega}$ denotes hyperbolic distance in $\Omega$; see Jør56. Then in 1984 Gaven Martin demonstrated that if $B$ is a ball (in $\mathrm{R}^{n}$ ) and $B \subset \Omega$, then $B$ is $k$-convex in $\Omega$ where $k=k_{\Omega}$ denotes quasihyperbolic distance in $\Omega$; see Mar85. We establish similar results for the Ferrand and Kulkarni-Pinkhall metrics; see 2.C.3 for their definitions.

Let $\mathcal{M}=\left\{\rho_{\Omega} d s\right\}_{\Omega \in \mathcal{O}}$ be a class of conformal metrics defined on domains $\Omega$ in some collection $\mathcal{O}$ of domains in $\hat{\mathrm{R}}^{n}$; see $\$ 2 . \mathrm{C}$ A non-empty open set $U \subset \hat{\mathrm{R}}^{n}$ is universally convex with respect to $\mathcal{M}$ (or briefly, $\rho$-UC), provided

$$
U \subset \Omega \in \mathcal{O} \Longrightarrow U \text { is convex in } \Omega_{\rho} \text {; }
$$

Received by the editors November 10, 2015 and, in revised form, June 23, 2016.

2010 Mathematics Subject Classification. Primary 53A30; Secondary 53C22, 51F99, 30C65, $30 \mathrm{~F} 45$

Key words and phrases. Geodesic convexity, quasihyperbolic metric, Ferrand metric, KulkarniPinkall metric, conformal metrics. 
we usually ignore trivial cases such as $U=\hat{\mathrm{R}}^{n}, U=\mathrm{R}^{n}$, etc. The results of Jørgensen and Martin mentioned above can now be stated as follows:

Disks (in $\hat{\mathrm{C}}$ ) are universally hyperbolically convex.

Balls (in $\mathrm{R}^{n}$ ) are universally quasihyperbolically convex.

In 1982 Barbara Brown Flinn [Fli83] showed that disks (in C) are the only (nontrivial) universally hyperbolically convex objects. Here is our main result. (See 2.C.3, \$2.C.2 $2 . \mathrm{D}$ for the definitions of $\varphi d s, \mu d s, \tau d s$ and $\mathcal{F}, \mathcal{K} \mathcal{P}, \mathcal{T}$.)

Theorem A. Let $n \geq 2$. For a non-trivial non-empty open $U \subset \hat{\mathrm{R}}^{n}$, these are equivalent:

(1) $U$ is universally convex with respect to the class $\mathcal{F}:=\left\{\varphi_{\Omega} d s\right\}_{\Omega \in \mathcal{Q}}$.

(2) $U$ is universally convex with respect to the class $\mathcal{K} \mathcal{P}:=\left\{\mu_{\Omega} d s\right\}_{\Omega \in \mathcal{Q}}$.

(3) $U$ is universally convex with respect to the class $\mathcal{T}:=\left\{\tau_{a b} d s\right\}_{a \neq b}$.

(4) $U$ is an open ball in $\hat{\mathrm{R}}^{n}$.

By a sphere in $\hat{\mathrm{R}}^{n}$ we mean any $\mathrm{S}^{k}(a ; r) \subset \mathrm{R}^{n}$ or the $\hat{\mathrm{R}}^{n}$-closure of any affine subspace of $\mathrm{R}^{n}$. A non-empty subset $A$ of a sphere $S$ in $\hat{\mathrm{R}}^{n}$ is declared to be circle convex in $S$ provided $A \cap C$ is connected for each circle $C$ in $S$. Evidently, circle convexity is a Möbius invariant, and any $A \subset \mathrm{R}^{n}$ that is circle convex in $\hat{\mathrm{R}}^{n}$ is a priori Euclidean convex. The main tool we use to prove Theorem $\mathrm{A}$ is the following characterization of closed circle convex sets.

Proposition B. Let $n \geq 2$. A non-empty closed subset $A$ of $\hat{\mathrm{R}}^{n}$ is circle convex in $\hat{\mathrm{R}}^{n}$ if and only if either $A=\hat{\mathrm{R}}^{n}$, or $A=\{p\}$ for some $p \in \hat{\mathrm{R}}^{n}$, or $A$ is a closed ball in $\hat{\mathrm{R}}^{n}$.

In fact, the above is but a special case of the following.

Theorem C. Let $n \geq 2$. For any non-empty subset $A$ of $\hat{\mathrm{R}}^{n}$, the following are equivalent:

(1) A is circle convex in $\hat{\mathrm{R}}^{n}$.

(2) For any Möbius transformation $\hat{\mathrm{R}}^{n} \stackrel{T}{\rightarrow} \hat{\mathrm{R}}^{n}$ with $T(A) \subset \mathrm{R}^{n}, T(A)$ is Euclidean convex.

(3) Either $A=\hat{\mathrm{R}}^{n}$, or $A=\hat{\mathrm{R}}^{n} \backslash\{p\}$ for some $p$, or $A=\{p\}$ for some $p$, or $A=B \cup C$ where $B$ is an open ball in $\hat{\mathrm{R}}^{n}, C \subset \partial B$, and either $C=\emptyset$ or if $n>2$, then $C$ is circle convex in $\partial B$, and if $n=2$, then $C$ is connected $\oplus$

Here is a noteworthy consequence of the proof of Theorem $\mathrm{A}$.

Corollary D. Let $\mathcal{M}=\left\{\rho_{\Omega} d s\right\}_{\Omega \in \mathcal{O}}$ be a Möbius invariant class of conformal metrics with $\mathrm{R}^{n} \backslash\{0\} \in \mathcal{O}$. Suppose $U$ is non-trivial and universally convex with respect to $\mathcal{M}$. Then $U$ is an open ball in $\hat{\mathrm{R}}^{n}$.

A natural question to ask is whether or not Möbius balls are universally convex with respect to any Möbius invariant class of conformal metrics. In contrast to [Mar85, Cor. 2.10] (where this is claimed to be trueף), we have the following.

\footnotetext{
*Here by non-trivial we simply mean that $U$ is not the entire sphere nor a once or twice punctured sphere.

†So, $C=\partial B$, or $C$ is a subarc of $\partial B$, or $C$ is a single point, or $C=\emptyset$.

${ }^{\ddagger}$ Gaven's readers should have been more careful!
} 
Example E. There are large Möbius invariant classes $\mathcal{M}=\left\{\rho_{\Omega} d s\right\}_{\Omega \in \mathcal{O}}$ of complete conformal metrics with the property that

$\forall \Omega \in \mathcal{O}, \forall r>0, \exists$ balls in $\Omega$ with radius at most $r$ which are not $\rho$-convex .

Here is another question the reader is surely pondering: What about universal quasihyperbolic convexity? In addition to balls being $k$-UC (Martin's result), it is straightforward to show that Euclidean half-spaces are $k$-UC. In fact, an unbounded (non-trivial) open subset of $\mathrm{R}^{n}$ is $k$-UC if and only if it is a half-space. In general, non-trivial $k$-UC sets are convex, however, numerical experiments suggest that fat plane ellipses may be $k$-UC.

We prove the above assertions (Theorem A, Proposition B, Theorem C, Corollary (D) in Section 3 and discuss Example Ein Section 4. Section 2 contains standard information including basic definitions, notation, and some results of independent interest such as Corollary 2.6 (which characterizes all Möbius invariant conformal metrics on doubly punctured spheres), Proposition 2.3 (which presents a useful formula relating any two inversions in terms of two natural reflections), and Proposition 2.11 (which establishes the expansion of diameter and circumdiameter under inversion).

\section{Preliminaries}

2.A. Basic information. Our notation is relatively standard. We write $|x-y|$ for the Euclidean distance between points $x, y$ in Euclidean space $\mathrm{R}^{n} ; \mathrm{B}^{n}(x ; r), \mathrm{B}^{n}[x ; r]$, and $\mathrm{S}^{n-1}(x ; r)$ are the open Euclidean ball, closed Euclidean ball, and Euclidean sphere (respectively) of radius $r$ and centered at the point $x$. Also, $\mathrm{B}^{n}:=\mathrm{B}^{n}(0 ; 1)$ and $S^{n-1}:=S^{n-1}(0 ; 1)$ are the open unit ball and unit sphere, and $H^{n}$ is the open upper half-space. The standard unit basis vectors are $\mathbf{e}_{1}, \ldots, \mathbf{e}_{n}$.

$\mathrm{A}(\mathrm{n})$ (affine) $k$-plane in $\mathrm{R}^{n}$, with $k \in\{1, \ldots, n-1\}$, is a $k$-dimensional vector (affine) subspace of $\mathrm{R}^{n}$. Thus a hyperplane in $\mathrm{R}^{n}$ is an affine $(n-1)$-plane.

We denote the $n$-sphere by $\hat{\mathrm{R}}^{n}:=\mathrm{R}^{n} \cup\{\infty\}$ and remind the reader that an open Möbius ball (i.e., an open ball in $\hat{\mathrm{R}}^{n}$ ) is either an open Euclidean ball, an open Euclidean half-space, or the complement (with respect to $\hat{\mathrm{R}}^{n}$ ) of a closed Euclidean ball; similarly for spheres and circles in $\hat{\mathrm{R}}^{n}$. All topology is with respect to $\hat{\mathrm{R}}^{n}$, so every closed set in $\hat{\mathrm{R}}^{n}$ is compact.

Almost everywhere, $\Omega$ is a quasihyperbolic domain $\Omega$ in $\hat{\mathrm{R}}^{n}$. Once, twice, and thrice punctured spheres are denoted by

$$
\hat{\mathrm{R}}_{a}^{n}:=\hat{\mathrm{R}} \backslash\{a\}, \quad \hat{\mathrm{R}}_{a b}^{n}:=\hat{\mathrm{R}}^{n} \backslash\{a, b\}, \quad \hat{\mathrm{R}}_{a b c}^{n}:=\hat{\mathrm{R}}^{n} \backslash\{a, b, c\}
$$

and similarly

$$
\mathrm{R}_{a b}^{n}:=\hat{\mathrm{R}}_{a b \infty}^{n}, \quad \mathrm{R}_{a}^{n}:=\hat{\mathrm{R}}_{a \infty}^{n}, \quad \mathrm{R}_{\star}^{n}:=\mathrm{R}_{0}^{n}=\mathrm{R}^{n} \backslash\{0\} ;
$$

the above definitions are for distinct points $a, b, c$ in $\hat{\mathrm{R}}^{n}$.

The quantity $\delta(x)=\delta_{\Omega}(x):=\operatorname{dist}(x, \partial \Omega)=\operatorname{dist}\left(x, \Omega^{c} \cap \mathrm{R}^{n}\right)$ is the Euclidean distance from $x \in \mathrm{R}^{n}$ to the boundary of $\Omega$, and when $\Omega \subset \mathrm{R}^{n}, 1 / \delta$ is the metricdensity for the so-called quasihyperbolic metric $|d x| / \delta(x)$; see $\$ 2 . \mathrm{C} .2$ We make frequent use of the notation

$$
\mathrm{B}(x)=\mathrm{B}_{\Omega}(x):=\mathrm{B}^{n}\left(x ; \delta_{\Omega}(x)\right):=\mathrm{B}^{n}(x ; \delta(x))
$$

$\S$ Meaning that $\Omega^{c}:=\hat{\mathrm{R}}^{n} \backslash \Omega$ contains at least 2 points. 
for the maximal Euclidean balls in $\Omega$ centered at $x$; also, $\mathrm{B}[x]:=\mathrm{B}^{n}[x ; \delta(x)]$.

As our notation suggests, we do not include $\Omega$ whenever the region is clear from context. Often, if there are two regions in consideration, say $\Omega$ and $\Omega^{\prime}$, we will use a prime $\left(^{\prime}\right)$ to indicate quantities associated with $\Omega^{\prime}$. For example, $\delta^{\prime}(x)=\delta_{\Omega^{\prime}}(x)$.

2.B. Möbius transformations. We refer to [Bea83 for the definition and basic properties of Möbius transformations. We introduce the following notation:

$$
J(x):=x^{\star}:=\frac{x}{|x|^{2}}, \quad \text { and then for each } p \in \mathrm{R}^{n}, \quad J_{p}(x):=J(x-p)=(x-p)^{\star} .
$$

We remind the reader that the inversion $J$ can be viewed as reflection across the unit sphere. However, the inversion $J_{p}$ is the translation $x \mapsto x-p$ followed by reflection across the unit sphere. Thus, e.g., $J^{-1}=J$ whereas $J_{p}^{-1}(y)=y^{\star}+p$.

Using the Law of Cosines one can easily establish the useful fact that

$$
\left|x^{\star}-y^{\star}\right|=\frac{|x-y|}{|x||y|} .
$$

It is straightforward to compute the derivative of $J$; we record the fact that

$$
\left|J^{\prime}(x)\right|=|x|^{2} ;
$$

see, for example, Bea83, pp. 26,27].

We write $\operatorname{Möb}(\Omega)$ for the conformal automorphism group of $\Omega$; that is,

$\operatorname{Möb}(\Omega):=\left\{T \mid \hat{\mathrm{R}}^{n} \stackrel{T}{\rightarrow} \hat{\mathrm{R}}^{n}\right.$ is a Möbius transformation with $\left.T(\Omega)=\Omega\right\}$.

We follow the standard convention that in dimension $n=2$ we also require Möbius transformations to be orientation preserving. We note that for "most" domains $\Omega$, $\operatorname{Möb}(\Omega)$ is trivial; see $\mathrm{BM} 08$ and the many references therein.

As simple examples, we mention the following automorphism groups; see Bea83. Theorems 3.1.3, 3.1.5, 3.4.1, 3.5.1].

- $\operatorname{Möb}\left(\mathrm{R}^{n}\right)$ is just the group of similarities of $\mathrm{R}^{n}$ : $T \in \operatorname{Möb}\left(\mathrm{R}^{n}\right) \Longrightarrow \exists U \in O(n), r>0, b \in \mathrm{R}^{n}$ such that $T=r U+b$.

- $\operatorname{Möb}\left(\mathrm{B}^{n} \backslash\{0\}\right)=O(n)$ (the standard orthogonal group).

- $\operatorname{Möb}\left(\mathrm{R}_{\star}^{n}\right)$ is generated by "rotations", dilations, and $J$ :

$T \in \operatorname{Möb}\left(\mathrm{R}_{\star}^{n}\right) \Longrightarrow \exists U \in O(n), r>0$ such that either $T=r U$ or $T=r U \circ J$.

It is also not difficult to determine the automorphism groups for spherical rings (such as $\{1 / 2<|x|<2\}$ ) and for infinite slabs (such as $\left\{\left(x_{1}, \ldots, x_{n}\right)|| x_{n} \mid<1\right\}$ ).

Next we provide the nifty formula (2.4) that reveals an interesting relation between any two inversions $J_{p}$ and $J_{q}$. We employ this, together with Proposition 2.11. to give a geometric proof that Möbius balls are universally Ferrand convex and universally Kulkarni-Pinkall convex.

We require some notation; see Figure 1. Let $p, q$ be distinct points in $\mathrm{R}^{n}$, let $\mathrm{L}$ be the extended line through $p$ and $q$, and let $\mathrm{M}$ be the perpendicular bisector of $[p, q]$; thus $\mathrm{L}$ is orthogonal to the hyperplane $\mathrm{M}$ which passes through the midpoint $m:=(p+q) / 2$. Next, let $\mathrm{P}$ be the image of $\mathrm{M}$ under the translation $x \mapsto x-m$; so $P$ is parallel to $M$ and passes through the origin. Notice that (by symmetry) the $J_{p}, J_{q}$ images of $\mathrm{M}$ are spheres with centers $J_{p}(q), J_{q}(p)$ (respectively) and both of these spheres are tangent to $\mathrm{P}$ at the origin. 


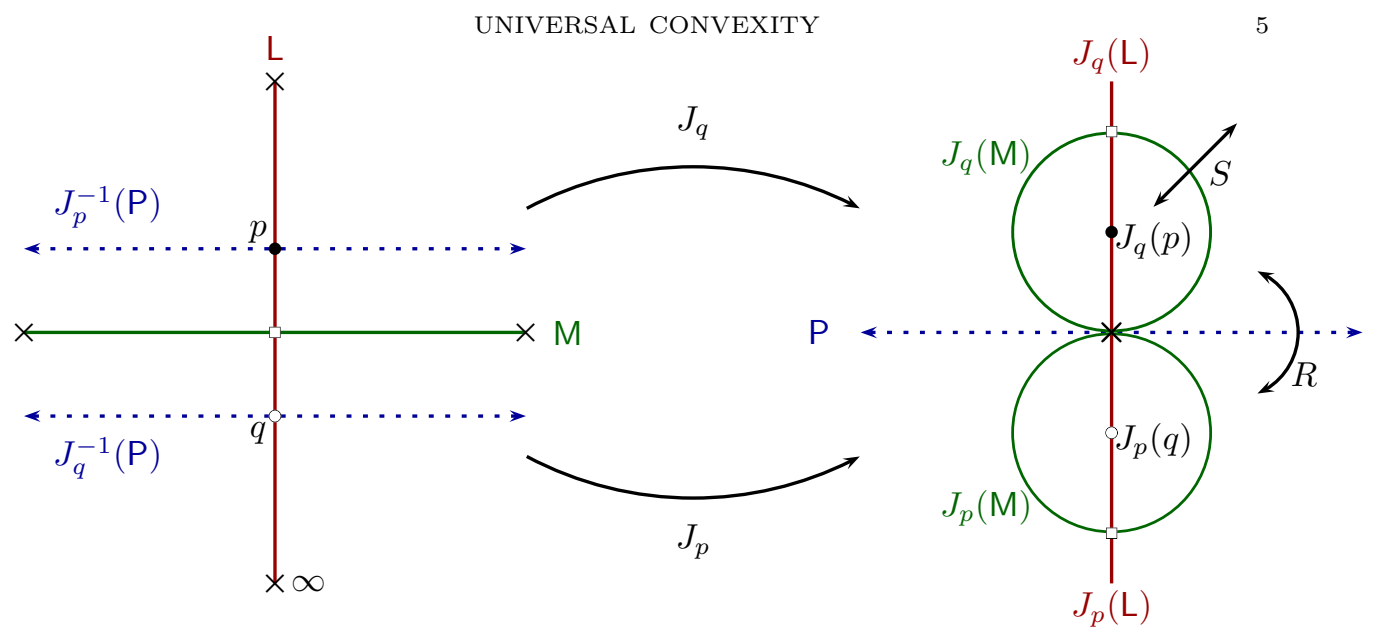

Figure 1. The hyperplane $\mathrm{M}$ and its $J_{p}$ and $J_{q}$ images

2.3. Proposition. Let $p, q$ and $\mathrm{M}, \mathrm{P}$ be as described above. Let $R$ and $S$ denote the reflections across $\mathrm{P}$ and $J_{q}(\mathrm{M})$ respectively. Then

$$
R \circ J_{p}=S \circ J_{q}, \quad \text { so, e.g., } J_{q}=S \circ R \circ J_{p} .
$$

Proof. We claim that the Möbius transformation $T:=R \circ J_{p} \circ J_{q}^{-1}$ pointwise fixes the sphere $J_{q}(\mathrm{M})$. Since $T(\infty)=R\left(J_{p}(q)\right)=J_{q}(p) \neq \infty$, it follows from [Bea83, Theorem 3.2.4] that $T=S$, which gives (2.4).

We verify that for each $x \in \mathrm{M}, R\left(J_{p}(x)\right)=J_{q}(x)$. This is trivial for the point at infinity, so, fix $x \in \mathrm{M} \cap \mathrm{R}^{n}$. It is geometrically transparent (and straightforward to verify analytically that $x-p$ and $x-q$ are symmetric with respect to $\mathrm{P}$. In particular,

$$
\text { for any } s>0, \quad R(s(x-p))=s(x-q) \text {. }
$$

As $x \in \mathrm{M} \cap \mathrm{R}^{n},|x-p|=|x-q|$, and so taking $s:=1 /|x-p|^{2}=1 /|x-q|^{2}$ we obtain

$$
R \circ J_{p}(x)=R\left((x-p)^{\star}\right)=R(s(x-p))=s(x-q)=(x-q)^{\star}=J_{q}(x)
$$

which establishes our claim.

There is a similar type result for any Apollonian sphere with limit point $p, q$.

2.C. Conformal metrics. A conformal metric on a region $\Omega \subset \mathrm{R}^{n}$ has the form $\rho d s=\rho(x)|d x|$ where $\rho$ is some positive Borel function defined on $\Omega \cap \mathrm{R}^{n}$ (with the property that the line element $\rho d s$ integrates to an honest distance function); we call such a $\rho$ a metric-density. For our purposes, we always assume that $\rho$ is in fact continuous. When $\Omega \subset \hat{\mathrm{R}}^{n}$ contains the point at infinity, we must use local coordinates and remember that we are dealing with a metric. Here we consider several such conformal metrics.

When two conformal metrics, say $\rho d s$ and $\sigma d s$, are both defined on some $\Omega$, their metric ratio $\rho d s / \sigma d s$ is a well-defined positive function on $\Omega$. We write $\rho \leq C \sigma$ to indicate that this metric ratio is bounded above by $C$.

\footnotetext{
【Indeed, since $p-q \perp \mathrm{M}, x-p+m$ and $x-q+m$ are symmetric with respect to $\mathrm{M}$.
} 
We recall that when $\Omega \stackrel{T}{\rightarrow} \Omega^{\prime}$ is (the restriction of) a Möbius transformation, so a conformal map, the pullback of a conformal metric $\rho^{\prime} d s=\rho^{\prime}(y)|d y|$ on $\Omega^{\prime}=T(\Omega)$ is the conformal metric $\rho d s=T^{\sharp}\left[\rho^{\prime} d s\right]=\rho(x)|d x|$ on $\Omega$ defined by

$$
\rho(x)|d x|=T^{\sharp}\left[\rho^{\prime}(y)|d y|\right]:=\rho^{\prime}(T(x))\left|T^{\prime}(x)\right||d x| .
$$

Note that the map $T$ is then an isometry between the metric spaces $\left(\Omega, d_{\rho}\right)$ and $\left(\Omega^{\prime}, d_{\rho^{\prime}}\right)$.

Many, but not all, of the metrics that we work with are defined on all quasihyperbolic domains and are Möbius invariant (so, conformally invariant when $n \geq 3$ ). A conformal metric $\rho d s$ on $\Omega$ is Möbius invariant provided for each automorphism $T \in \operatorname{Möb}(\Omega), T^{\sharp}[\rho d s]=\rho d s$. A family $\mathcal{O}$ of domains $\Omega$ is Möbius invariant if for each $\Omega$ in $\mathcal{O}$, every Möbius image $T(\Omega)$ also belongs to $\mathcal{O}$. A class $\left\{\rho_{\Omega} d s\right\}_{\Omega \in \mathcal{O}}$ of conformal metrics, each defined on a domain $\Omega$ in $\mathcal{O}$, is Möbius invariant if $\mathcal{O}$ is Möbius invariant and if the pullback metric $T^{\sharp}\left[\rho_{\Omega^{\prime}} d s\right]$ equals $\rho_{\Omega} d s$ (for each $\Omega, \Omega^{\prime} \in \mathcal{O}$ and each Möbius transformation $T$ with $T(\Omega)=\Omega^{\prime}$ ). See $\oint 2$.D for examples of Möbius invariant classes of metrics.

It is important to realize that the variety of Möbius invariant conformal metrics defined on a given domain depends strongly on the domain's automorphism group: when there are more automorphisms, there are fewer such metrics. For example, if say $\left\{\rho_{B} d s\right\}_{B \in \mathcal{B}}$ is a Möbius invariant class of conformal metrics defined on balls in $\hat{\mathrm{R}}^{n}$, then in fact there is a universal constant $C$ such that for each $B \in \mathcal{B}$, $\rho_{B} d s=C \lambda_{B} d s$ (see $\$ 2 . \mathrm{C} .1$ for the definition of $\lambda_{B} d s$ and $\$ 2 . \mathrm{D}$ for the description of $\mathcal{B}$ ). This is not difficult to establish; see HMM08, Lemma 4.1]. A similar result is described below in Lemma 2.5.

2.C.1. Hyperbolic metrics. Here we identify $\hat{\mathrm{R}}^{2}$ with the Riemann sphere $\hat{C}$. For a hyperbolic domain $\Omega \subset \hat{\mathrm{C}}$ (which has at least three boundary points), there exists a unique metric $\lambda d s=\lambda_{\Omega} d s$ on $\Omega$ which enjoys the property that its pullback $p^{\sharp}[\lambda d s]$, with respect to any holomorphic universal covering projection $p: \mathrm{D} \rightarrow \Omega$, is the hyperbolic metric $\lambda_{\mathrm{D}}(\zeta)|d \zeta|=2\left(1-|\zeta|^{2}\right)^{-1}|d \zeta|$ on the unit disk D. In terms of such a covering $p$, the metric-density $\lambda=\lambda_{\Omega}$ of the Poincaré hyperbolic metric $\lambda_{\Omega}(z)|d z|$ can be determined from

$$
\lambda(z)=\lambda_{\Omega}(z)=\lambda_{\Omega}(p(\zeta)):=2\left(1-|\zeta|^{2}\right)^{-1}\left|p^{\prime}(\zeta)\right|^{-1} ;
$$

of course this is only valid at points $z=p(\zeta) \in \Omega \cap \mathrm{C}$. Yet another description is that $\lambda d s$ is the maximal complete constant curvature -1 metric on $\Omega$.

There is no concept of a hyperbolic metric on a general domain in $\hat{\mathrm{R}}^{n}$, but each Möbius ball in $\hat{\mathrm{R}}^{n}$ does carry a (real) hyperbolic metric. For example, the hyperbolic metric on $\mathrm{B}^{n}(a ; r)$ is just $\left[2 r /\left(r^{2}-|x-a|^{2}\right)\right]|d x|$.

2.C.2. Quasihyperbolic metrics. As alluded to above, for proper subdomains $\Omega \subsetneq$ $\mathrm{R}^{n}$, the so-called quasihyperbolic metric is given by $|d x| / \delta(x)$. This metric has proven useful in many areas of geometric analysis. For example, the quasihyperbolic metric in the punctured space $\mathrm{R}_{*}^{n}$ is simply $|d x| /|x|$, and the quasihyperbolic metric on any half-space is precisely its hyperbolic metric.

A Möbius invariant analog of the metric $|d x| /|x|$ can be defined in the twice punctured sphere $\hat{\mathrm{R}}_{a b}^{n}$ by

$$
\tau_{a b}(x)|d x|:=\frac{|a-b|}{|x-a||x-b|}|d x|,
$$


with the standard interpretation if one of $a$ or $b$ is the point at infinity. For example, $\tau_{a \infty}(x)|d x|=|d x| /|x-a|$, which is the quasihyperbolic metric on the punctured space $\mathrm{R}_{a}^{n}$.

It is straightforward to check that the metrics $\tau_{a b} d s$ are Möbius invariant. In fact, $\tau_{a b} d s$ is the unique metric on $\hat{\mathrm{R}}_{a b}^{n}$ with the property that for any Möbius transformation $T$ with $T\left(\hat{\mathrm{R}}_{a b}^{n}\right)=\mathrm{R}_{*}^{n}, \tau_{a b}(x)|d x|=T^{\sharp}[|d y| /|y|]$. See [HJ13, §2.D.1].

Because of the above, it seems appropriate to dub $\tau_{a b} d s$ the quasihyperbolic metric on $\hat{\mathrm{R}}_{a b}^{n}$.

Similar to Möbius balls, twice punctured spheres support an essentially unique Möbius invariant metric. First we establish this for the standard twice punctured sphere.

2.5. Lemma. A conformal metric $\rho$ ds on $\mathrm{R}_{\star}^{n}$ is Möbius invariant if and only if there is a positive constant $C=C(\rho)$ such that $\rho d s=C|d x| /|x|$. In fact, $C:=\rho(e)$ for any $e \in \mathrm{S}^{n-1}$.

Proof. Using the fact that $\mathrm{Möb}\left(\mathrm{R}_{\star}^{n}\right)$ consists of "rotations", dilations, and inversion with respect to the origin, it is straightforward to show that $|d x| /|x|$ is Möbius invariant. (To check that this metric is invariant with respect to the inversion $J(x):=x^{\star}:=x /|x|^{2}$, it is useful to use the facts that $J^{\prime}(x)=|x|^{-2}\left(I-2 Q_{x}\right)$ where $I$ is the identity and $\left[Q_{x}\right]_{i j}:=x_{i} x_{j} /|x|^{2}$, and that $I-2 Q_{x}$ is orthogonal. See [Bea83, pp. 25-26].)

For the converse, suppose $\rho d s$ is a Möbius invariant conformal metric on $\mathrm{R}_{\star}^{n}$. Put $C:=\rho\left(\mathbf{e}_{1}\right)$. Let $a \in \mathrm{R}_{\star}^{n}$. Pick any $R \in O(n)$ with $R(a /|a|)=\mathbf{e}_{1}$ and define $T(x):=R(x /|a|)$. Then $T^{\prime}(x)=R^{\prime}(x /|a|)|a|^{-1}$, so by Möbius invariance

$$
\rho(x)|d x|=T^{\sharp}\left[\rho(y)|d y|=\rho(T(a))\left|T^{\prime}(x)\right||d x|\right]
$$

and evaluating at $x=a$ gives

$$
\rho(a)=\rho\left(\mathbf{e}_{1}\right)\left|T^{\prime}(a)\right|=C /|a|
$$

as asserted.

2.6. Corollary. Suppose $\left\{\rho_{a b} d s\right\}_{a \neq b}$ is a Möbius invariant class of conformal metrics defined on twice punctured spheres $\hat{\mathrm{R}}_{a b}^{n}$. Then there is a constant $C=C\left(\rho_{0 \infty}\right)$ such that for all distinct points $a, b \in \hat{\mathrm{R}}^{n}, \rho_{a b} d s=C \tau_{a b} d s$.

2.C.3. Ferrand and Kulkarni-Pinkhall metrics. These metrics, denoted by $\varphi d s$ and $\mu d s$, respectively, are defined on each quasihyperbolic domain $\Omega$ in $\hat{\mathrm{R}}^{n}$.

The Ferrand metric $\varphi d s=\varphi_{\Omega} d s$ on $\Omega$ can be defined for points $x \in \Omega \cap \mathrm{R}^{n}$ by

$$
\varphi(x)=\varphi_{\Omega}(x):=\sup _{a, b \in \Omega^{c}} \frac{|a-b|}{|x-a||x-b|} .
$$

This metric was first introduced by Jacqueline Ferrand in Fer88.

The Kulkarni-Pinkall metric $\mu d s=\mu_{\Omega} d s$ on $\Omega$ can be defined, for points $x \in$ $\Omega \cap \mathrm{R}^{n}$, by

$$
\mu(x)=\mu_{\Omega}(x):=\inf \left\{\lambda_{B}(x) \mid x \in B \subset \Omega, B \text { a Möbius ball }\right\} .
$$

This metric was first introduced by Ravi Kulkarni and Ulrich Pinkall in [KP94]. The above definition was first presented in HMM03. 
These metrics are bilipschitz equivalent to each other, and bilipschitz equivalent to the quasihyperbolic metric (on finite domains). In fact, we have

$$
\mu \leq\left(\frac{2 n}{n+1}\right)^{1 / 2} \varphi \text { and } \frac{1}{\delta} \leq \varphi \leq \mu \leq \frac{2}{\delta}
$$

where the $\delta^{-1} \leq \varphi$ inequality holds provided $\Omega \subset \mathrm{R}^{n}$; see (2.10), and also, HIM08, Cor. 4.6] and HMM05].

We note that the Ferrand, Kulkarni-Pinkall, and hyperbolic metrics all agree in any Möbius ball $B$; i.e., $\varphi_{B}=\lambda_{B}=\mu_{B}$. That Ferrand's metric is Möbius invariant follows from the Möbius invariance of $\tau_{a b} d s$. That the Kulkarni-Pinkall metric is Möbius invariant follows from its definition along with the Möbius invariance of the hyperbolic metric.

It is not difficult to show that the Ferrand and Kulkarni-Pinkall extremal quantities are achieved. For any quasihyperbolic $\Omega$ and any $x \in \Omega \cap \mathrm{R}^{n}$, there are points $a, b \in \partial \Omega$ such that $\varphi(x)=\tau_{a b}(x)$; e.g., see [HJ13, Theorem 3.1, Remarks 3.9]. Similarly, for any quasihyperbolic $\Omega$ and any $x \in \Omega \cap \mathrm{R}^{n}$, there is a Möbius ball $P=\mathrm{P}(x)=\mathrm{P}_{\Omega}(x)$ (that we call the Kulkarni-Pinkall extremal ball for $x$ in $\Omega$ ) with $x \in P \subset \Omega$ such that $\mu(x)=\lambda_{P}(x)$. Moreover, $\partial P \cap \partial \Omega$ contains at least two points and $P$ is in fact the Kulkarni-Pinkall extremal ball for each point in the closed hyperbolically convex hull $K=\mathrm{K}(x)$ of $\partial P \cap \partial \Omega \subset P$. See HMM03, Theorems 3.5,4.1,4.6] for a proof of this in dimension $n=2$; also, see HIM08, Prop. 4.4, Cor 4.5].

To be precise, given a Möbius ball $B \subset \hat{\mathrm{R}}^{n}$ and a non-empty set $A \subset \bar{B}$, we write $\mathrm{K}_{B}(A)$ to denote the smallest set $K$ that is closed in $B$, that is hyperbolically convex (i.e., convex with respect to hyperbolic distance in $B$ ), and whose closure with respect to $\hat{\mathrm{R}}^{n}$ contains $A$. Thus, $\mathrm{K}_{B}(A)$ is the intersection of all sets $H$ where $H \subset B$ is a closed hyperbolic half-space in $B$ with $\bar{H} \supset A$. With this notation, for each $x \in \Omega, \mathrm{K}(x):=\mathrm{K}_{\mathrm{P}(x)}(\partial \mathrm{P}(x) \cap \partial \Omega)$.

2.D. Classes of metrics. Here we present some elementary examples of classes of conformal metrics. We consider the following collections of domains in $\hat{\mathrm{R}}^{n}$ :

- $\mathcal{B}$ is the set of all Möbius balls in $\hat{\mathrm{R}}^{n}$,

- $\mathcal{D}$ is the set of all quasihyperbolic domains $\Omega \subsetneq \mathrm{R}^{n}$,

- $\mathcal{Q}$ is the set of all quasihyperbolic domains in $\hat{\mathrm{R}}^{n}$.

The conformal metric classes mentioned in Theorem $\mathrm{A}$ are described below; all of these, except $\mathcal{K}$ (which is affine invariant), are Möbius invariant:

- $\mathcal{H}:=\left\{\lambda_{B} d s \mid B \in \mathcal{B}\right\}$ is the class of all hyperbolic metrics,

- $\mathcal{K}:=\left\{\delta_{\Omega}^{-1} d s \mid \Omega \in \mathcal{D}\right\}$ is the class of all quasihyperbolic metrics,

- $\mathcal{F}:=\left\{\varphi_{\Omega} d s \mid \Omega \in \mathcal{Q}\right\}$ is the class of all Ferrand metrics,

- $\mathcal{K P}:=\left\{\mu_{\Omega} d s \mid \Omega \in \mathcal{Q}\right\}$ is the class of all Kulkarni-Pinkall metrics,

- $\mathcal{T}:=\left\{\tau_{a b} d s \mid a \neq b\right.$ points in $\left.\hat{\mathrm{R}}^{n}\right\}$ is the class of all two-point quasihyperbolic metrics defined on twice punctured spheres $\hat{\mathrm{R}}_{a b}^{n}$.

In dimension $n=2$ we enlarge the class $\mathcal{H}$ by considering all hyperbolic domains in $\hat{C}$.

It is important to realize that in parts (1) and (2) of Theorem $\mathrm{A} \mathcal{Q}$ is the collection of domains being considered whereas in part (3) it is just the smaller collection of all twice punctured spheres. 
2.E. Circumballs and circumdiameters. For any non-empty bounded set $A \subset$ $\mathrm{R}^{n}$, there is a unique smallest closed (Euclidean) ball $\mathrm{B}_{A}$ that contains $A$; we call $\mathrm{B}_{A}$ the circumball about $A$. Then the circumdiameter of $A$ is

$$
\operatorname{cdiam}(A):=\operatorname{diam}\left(\mathrm{B}_{A}\right) \text {. }
$$

Jung's theorem (see Ber87, 11.5.8, p. 357]) provides the following information about circumballs.

2.9. Fact. For any non-empty bounded set $A \subset \mathrm{R}^{n}$ :

(a) The center of $\mathrm{B}_{A}$ belongs to the convex hull of $\bar{A} \cap \partial \mathrm{B}_{A}$.

(b) $\operatorname{diam}(A) \leq \operatorname{diam}\left(\mathrm{B}_{A}\right) \leq(2 n /(n+1))^{1 / 2} \operatorname{diam}(A)$.

Now we present a method for calculating the Ferrand and Kulkarni-Pinkall metrics that is based on Euclidean diameters and circumdiameters. (This provides some evidence that there is a strong connection between the Ferrand and KulkarniPinkall metrics!) For each $x \in \Omega \cap \mathrm{R}^{n}\left(\Omega\right.$ a quasihyperbolic domain in $\left.\hat{\mathrm{R}}^{n}\right)$,

$$
\varphi(x)=\operatorname{diam} J_{x}\left(\Omega^{c}\right) \quad \text { and } \quad \mu(x)=\operatorname{cdiam} J_{x}\left(\Omega^{c}\right) ;
$$

see [HJ13, 3.3(a)] and [HIM08, Prop. 4.4]. In fact, the Kulkarni-Pinkall extremal balls are given via

$$
\mathrm{P}(x)=J_{x}^{-1}\left[\left(\mathrm{~B}_{J_{x}\left(\Omega^{c}\right)}\right)^{c}\right] .
$$

Because of (2.10), it is important to understand how inversions distort diameters and circumdiameters. It is geometrically transparent that the inversion $J(x)=$ $x^{\star}:=x /|x|^{2}$ is expanding inside the unit ball, and thus increases diameters; e.g., see (2.2). The same holds for circumdiameters, which may be well known but is difficult to locate in the literature.

2.11. Proposition. Let $E$ be a non-degenerat 1 ll compact subset of some open Euclidean ball. Let $E^{\star}$ denote the reflection of $E$ across the boundary of this ball. Then

$$
\operatorname{diam}\left(E^{\star}\right)>\operatorname{diam}(E)
$$

and

$$
\operatorname{cdiam}\left(E^{\star}\right)>\operatorname{cdiam}(E) .
$$

Proof. By using a similarity transformation, we may assume that $\mathrm{B}^{n}$ is the open ball containing $E$. The diameter expansion is nearly trivial. Pick $x, y \in E$ with $\operatorname{diam}(E)=|x-y|$. Then by (2.1),

$$
\operatorname{diam}\left(E^{\star}\right) \geq\left|x^{\star}-y^{\star}\right|=\frac{|x-y|}{|x||y|}>|x-y|=\operatorname{diam}(E)
$$

where the strict inequality holds because $x, y \in \mathrm{B}^{n}$. Thus (2.11a) holds.

To establish (2.11b), let $A:=\mathrm{B}_{E^{\star}}=\mathrm{B}^{n}[a ; r]$ be the circumball containing $E^{\star}$. Recall that the center $a$ of $A$ lies in the convex hull of $E^{\star} \cap \partial A$; see Fact 2.9(a). Since $E$ is compact and in $\mathrm{B}^{n}, \operatorname{cdiam}(E)<2$. Thus we may assume that $2 r=$ $\operatorname{diam}(A)=\operatorname{cdiam}\left(E^{\star}\right)<2$.

Note that as $A \backslash \overline{\mathrm{B}}^{n} \supset E^{\star} \neq \emptyset, r>1-|a|$ so $|a|>1-r$. (In fact, $|a|^{2}>1-r^{2}$.)

If $A \cap \overline{\mathrm{B}}^{n}=\emptyset$, then $A^{\star}:=J(A) \subset \mathrm{B}^{n}$, and so by (2.11a)

$$
\operatorname{cdiam}(E) \leq \operatorname{diam}\left(A^{\star}\right)<\operatorname{diam}(A)=\operatorname{cdiam}\left(E^{\star}\right) .
$$

\footnotetext{
${ }$ Meaning that $E$ contains at least two points.
} 
Thus we may (even) assume that $A \cap \mathrm{B}^{n} \neq \emptyset$. That is, $1-r<|a|<1+r$.

Let $S$ be the $(n-2)$-sphere $S:=A \cap \partial \mathrm{B}^{n}=\partial A \cap \partial \mathrm{B}^{n}=\partial A \cap \mathrm{S}^{n-1}$. Next, let $B$ be the closed Euclidean ball with the same center and radius as $S$. (E.g, if $|a|^{2}+r^{2}=1$, then $a$ would be the common center of $B$ and $S$, and so $B=A$.)

We examine the three cases where $J\left(A \backslash \overline{\mathrm{B}}^{n}\right) \supsetneq A \cap \mathrm{B}^{n}$ or $J\left(A \backslash \overline{\mathrm{B}}^{n}\right)=A \cap \mathrm{B}^{n}$ or $J\left(A \backslash \overline{\mathrm{B}}^{n}\right) \subsetneq A \cap \mathrm{B}^{n}$; equivalently, $|a|^{2}>1+r^{2}$ or $|a|^{2}=1+r^{2}$ or $|a|^{2}<1+r^{2}$, or in terms of the 'exterior' angle $\theta$ between $\partial A$ and $\mathrm{S}^{n-1}, 0<\theta<\pi / 2$ or $\theta=\pi / 2$ or $\pi / 2<\theta<\pi$. See Figure 2

Suppose $J\left(A \backslash \overline{\mathrm{B}}^{n}\right) \supsetneq A \cap \mathrm{B}^{n}$ (i.e., $|a|^{2}>1+r^{2}$ or $0<\theta<\pi / 2$ ). Let $x$ and $y$ be the points in $A$ that are, respectively, closest to and furthest from the origin. A glance at an appropriate diagram reveals that

$$
\left|y^{\star}\right|<|x|<|x|^{\star}<|y|
$$

so $|x||y|>1$ and therefore

$$
\operatorname{cdiam}(E) \leq \operatorname{diam}\left(A^{\star}\right)=\left|x^{\star}-y^{\star}\right|=\frac{|x-y|}{|x||y|}<|x-y|=\operatorname{diam}(A)=\operatorname{cdiam}\left(E^{\star}\right) .
$$

Suppose $J\left(A \backslash \overline{\mathrm{B}}^{n}\right)=A \cap \mathrm{B}^{n}$ (i.e., $|a|^{2}=1+r^{2}$ or $\left.\theta=\pi / 2\right)$. Here $A^{\star}=A$ so the above argument fails. In fact, the argument below (for the last case) also applies to this case, but the idea can be made more explicit here. Let $v$ denote the angle between the line segments $[0, a]$ and $[0, z]$ where $z$ is any point in $S$. Then (by looking at an appropriate picture) we see that

$$
\operatorname{diam}(B)=\operatorname{diam}(S)=2 \sin v=\frac{2 r}{|a|}=\frac{\operatorname{diam}(A)}{|a|}<\operatorname{diam}(A)
$$

and since $E \subset J\left(A \backslash \mathrm{B}^{n}\right) \subset B$, it now follows that

$$
\operatorname{cdiam}(E) \leq \operatorname{diam}(B)<\operatorname{diam}(A)=\operatorname{cdiam}\left(E^{\star}\right) .
$$

Suppose $J\left(A \backslash \overline{\mathrm{B}}^{n}\right) \subsetneq A \cap \mathrm{B}^{n}$ (i.e., $|a|^{2}<1+r^{2}$ or $\pi / 2<\theta<\pi$ ). Recall that the center $a$ of the circumball $A$ lies in the convex hull of $E^{\star} \cap \partial A$, and that $E^{\star} \subset A \backslash \overline{\mathrm{B}}^{n}$. In particular, this means that $a$ cannot be too close to the origin. For example, if $|a|^{2}<1-r^{2}$, then $B \supset A \backslash \mathrm{B}^{n} \supset E^{\star}$, but as $\operatorname{diam}(B)<\operatorname{diam}(A)$, this would contradict $A$ being the circumball about $E^{\star}$; therefore, $|a|^{2} \geq 1-r^{2}$. If $|a|^{2}=1-r^{2}$, then $B=A$, but as $E^{\star}$ is a compact subset of $A \backslash \overline{\mathrm{B}}^{n}$, the convex hull of $E^{\star} \cap \partial A$ would not contain $a$ which would give another contradiction. Thus, $|a|^{2}>1-r^{2}$, so $1-r^{2}<|a|^{2}<1+r^{2}$. (As alluded to above, here we could allow $|a|^{2}=1+r^{2}$.)

In this setting we have $B \supset A \cap \mathrm{B}^{n} \supset J\left(A \backslash \overline{\mathrm{B}}^{n}\right) \supset E$, and so

$$
\operatorname{cdiam}(E) \leq \operatorname{diam}(B)<\operatorname{diam}(A)=\operatorname{cdiam}\left(E^{\star}\right) .
$$

2.F. Miscellaneous facts. Here we provide some information that is used in the proofs of $(3) \Longrightarrow(4) \Longrightarrow(2)$ in Theorem A.

The following specialized fact provides an easy, albeit uninformative, analytic proof that Möbius balls are universally Kulkarni-Pinkall convex. Its significance is best understood by examining its role in the proof of $(4) \Longrightarrow(2)$ in Theorem $\mathrm{A}$.

2.12. Lemma. Let $\Omega \subset \hat{\mathrm{R}}^{n}$ be a quasihyperbolic domain. Suppose $\Omega$ contains a closed Möbius ball $\bar{H}$. Let $x \in \Omega \backslash \bar{H}$ and put $B:=\mathrm{P}(x)$ (the Kulkarni-Pinkall 


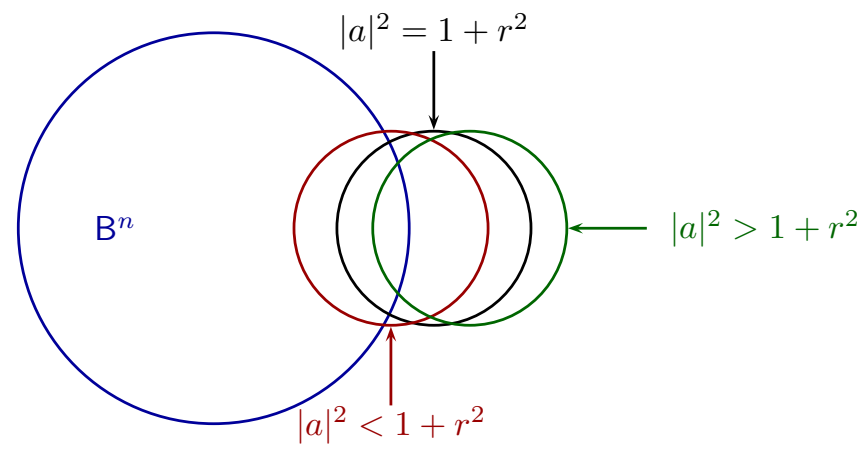

Figure 2. The balls $\mathrm{B}^{n}[a ; r]$

extremal ball for $x)$. Let $x^{*}$ and $B^{*}$ denote the reflections of $x$ and $B$ across $\partial H$, respectively. If

$$
B^{*} \not \subset \Omega \quad \text { or } \quad B^{*} \subset \Omega \text { and } B^{*}=\mathrm{P}\left(x^{*}\right)
$$

then

$$
x^{*} \in B \quad \text { and } \quad \lambda_{B}(x)>\lambda_{B}\left(x^{*}\right) .
$$

Proof. By applying a Möbius transformation, if necessary, we may assume that $H$ is the upper half-space $\mathrm{H}^{n}$; so, $x \in \mathrm{R}^{n} \backslash \overline{\mathrm{H}}^{n}$. We consider the two cases where $\partial B$ meets, or does not meet, $\partial \mathrm{H}^{n}$; in each case we examine subcases (and subsubcases, etc.) where $B$ is an open Euclidean ball or the complement of a closed Euclidean ball or an open half-space.

First, suppose that $\partial B \cap \partial \mathbf{H}^{n}=\emptyset$. Here there are only two subcases. Assume $B=\mathrm{B}^{n}(c ; r)$. Then necessarily, $B \subset \mathrm{R}^{n} \backslash \overline{\mathrm{H}}^{n}$, so $B^{*} \subset \mathrm{H}^{n} \subset \Omega$. It follows then that $B^{*}$ is not the Kulkarni-Pinkall extremal ball for any point in $\Omega$. So, our assertion holds.

Assume $B=\hat{\mathrm{R}}^{n} \backslash \mathrm{B}^{n}[c ; r]$. Then necessarily, $\mathrm{B}^{n}[c ; r] \subset \mathrm{R}^{n} \backslash \overline{\mathrm{H}}^{n}$, and $B \cup B^{*}=\hat{\mathrm{R}}^{n}$, so $B^{*} \not \subset \Omega$. Clearly, $x^{*} \in \mathrm{H}^{n} \subset B$, and as $|x-c|<\left|x^{*}-c\right|, \lambda_{B}(x)>\lambda_{B}\left(x^{*}\right)$.

Next, suppose that $\partial B \cap \partial \mathbf{H}^{n} \neq \emptyset$. Here we examine the cases where $\partial B$ is parallel to $\partial \mathrm{H}^{n}$, or where $\partial B$ is orthogonal to $\partial \mathrm{H}^{n}$, or where neither of these holds; in each case we consider $B$ an open Euclidean ball or the complement of a closed Euclidean ball or an open half-space. And then each of these many cases has further subcases, e.g., depending on where the 'center' of $B$ lies.

The cases where $\partial B$ and $\partial \mathrm{H}^{n}$ are parallel are straightforward and we leave their verification to the diligent reader.

Assume that $\partial B$ is orthogonal to $\partial \mathrm{H}^{n}$. Here we see that $B^{*}=B \subset \Omega$ and $x^{*} \in B$. However, since

$$
\partial B^{*} \cap \partial \Omega=\partial B \cap \partial \Omega \subset \partial B \backslash \overline{\mathbf{H}}^{n}
$$

it follows that

$$
\mathrm{K}_{B^{*}}\left(\partial B^{*} \cap \partial \Omega\right) \subset \mathrm{K}_{B}(\partial B \backslash \bar{H})=B \backslash \mathrm{H}^{n}
$$

which, in particular, means that $B^{*} \neq \mathrm{P}\left(x^{*}\right)$.

Finally, assume that $\partial B$ is neither parallel nor orthogonal to $\partial \mathrm{H}^{n}$. Here there are six subsubcases to consider (e.g. depending on where the 'center' of $B$ lies) 
most of which are straightforward. We examine the two interesting cases and leave verification of the remaining to the diligent reader. When $B=\mathrm{B}^{n}(c ; r)$ : if $c \in \mathrm{H}^{n}$, then $B^{*} \not \subset \Omega$ and $x^{*} \in B$ with $\lambda_{B}(x)>\lambda_{B}\left(x^{*}\right)$; if $c \notin \overline{\mathbf{H}}^{n}$, then $B^{*} \subset \Omega$ but $B^{*} \neq \mathrm{P}\left(x^{*}\right)$.

Suppose $B=\hat{\mathrm{R}}^{n} \backslash \mathrm{B}^{n}[c ; r]$. If $c \in \mathrm{H}^{n}$, then $B^{*} \subset \Omega$ but $\partial B^{*} \cap \partial \Omega=\emptyset$ so $B^{*} \neq \mathrm{P}(y)$ for any $y \in \Omega$. Assume $c \notin \overline{\mathrm{H}}^{n}$. Here both $B^{*} \subset \Omega$ or $B^{*} \not \subset \Omega$ are possible, and $B^{*}=\mathrm{P}\left(x^{*}\right)$ might hold too. In any event, $x^{*} \in B$ with $|x-c|<\left|x^{*}-c\right|$ so $\lambda_{B}(x)>\lambda_{B}\left(x^{*}\right)$.

Now suppose $B$ is an open Euclidean half-space. Recall that $\mathbf{e}_{n}$ is the inner unit normal for $\mathbf{H}^{n}$. Let $\mathbf{n}$ be the inner unit normal for $B$; so $\mathbf{n}$ is orthogonal to $\partial B$ and 'points' into $B$. When $\mathbf{e}_{n} \cdot \mathbf{n}>0, B^{*} \not \subset \Omega$ and $x^{*} \in B$ with $\lambda_{B}(x)>\lambda_{B}\left(x^{*}\right)$. Assume $\mathbf{e}_{n} \cdot \mathbf{n}<0$. Here

$$
B^{*} \subset \mathrm{H}^{n} \cup B \subset \Omega \quad \text { but } \quad \partial B^{*} \backslash \overline{\mathrm{H}}^{n} \subset B \subset \Omega, \quad \text { so } \partial B^{*} \cap \partial \Omega=\emptyset
$$

and therefore $B^{*}$ is not the Kulkarni-Pinkall extremal ball for any point in $\Omega$.

Next we establish the following technical result that we repeatedly use to do "dimension reduction" in our argument for $(3) \Longrightarrow(4)$ in Theorem A.

2.13. Lemma. Let $U$ be a non-empty open subset of the standard twice punctured sphere $\mathrm{R}_{\star}^{n}$; here $n \geq 2$. Suppose that $U$ is $k$-convex in $\mathrm{R}_{\star}^{n}$ and that either $(\mathrm{i}) \bar{U}=\hat{\mathrm{R}}^{n}$ or (ii) $\bar{U}=\overline{\mathrm{B}}^{n}$. Let $\mathrm{P}$ be an $(n-1)$-plane in $\mathrm{R}^{n}$ and put $V:=U \cap \mathrm{P}$. Then $V$ is non-empty, open in $\mathrm{P}, k$-convex in $\mathrm{P}_{\star}:=\mathrm{P} \backslash\{0\}$ and either (i) $\bar{V}=\overline{\mathrm{P}}:=\mathrm{P} \cup\{\infty\}$ or (ii) $\bar{V}=\overline{\mathrm{B}}^{n} \cap \mathrm{P}$, respectively.

Proof. Clearly, $V$ is open in $\mathrm{P}$. If $\gamma$ is a $k$-geodesic in $\mathrm{P}_{\star}$ with endpoints in $V$, then $\gamma$ is also a $k$-geodesic in $\mathrm{R}_{\star}^{n}$ with endpoints in $U$, so $\gamma$ lies in $U$ (and in $\mathrm{P}_{\star}$, so in $V)$.

We verify that $V$ is dense in either (i) $\overline{\mathrm{P}}$ or (ii) $\overline{\mathrm{B}}^{n} \cap \mathrm{P}$, respectively (and therefore, $V$ is non-empty). For this, it suffices to check that for every point $p$ in $\mathrm{P}_{\star}$ (or in $\mathrm{P}_{\star} \cap \mathrm{B}^{n}$ ) and all sufficiently small $r>0$,

$$
V \cap \mathrm{B}^{n}(p ; r) \neq \emptyset \quad\left(\text { or } V \cap \mathrm{B}^{n} \cap \mathrm{B}^{n}(p ; r) \neq \emptyset\right) .
$$

To this end, let $p \in \mathrm{P}_{\star}$ (or $p \in \mathrm{P}_{\star} \cap \mathrm{B}^{n}$ ) and $r \in(0,|p|)$ (with $r<1-|p|$ in the case $p \in \mathrm{P}_{\star} \cap \mathrm{B}^{n}$, so that $\left.\mathrm{B}^{n}(p ; r) \subset \mathrm{B}^{n}\right)$. Since $\mathrm{P}$ is a hyperplane, it separates $\mathrm{R}^{n}$ and so it also separates $\mathrm{B}^{n}(p ; r)$. Thus we can write $\mathrm{B}^{n}(p ; r) \backslash \mathrm{P}=A \cup B$ where $A$ and $B$ are non-empty disjoint open subsets of $\mathrm{R}^{n}$. Appealing to the hypothesis that $U$ is dense (either in $\hat{\mathrm{R}}^{n}$ or in $\bar{B}^{n}$ ), we find points $a \in A \cap U$ and $b \in B \cap U$.

Let $\gamma$ be a $k$-geodesic in $\mathrm{R}_{\star}^{n}$ with endpoints $a, b$. Since $U$ is $k$-convex in $\mathrm{R}_{\star}^{n}$, we know that $\gamma$ lies in $U$. By Martin's theorem, $\mathrm{B}^{n}(p ; r) \subset \mathrm{R}_{\star}^{n}$ is also $k$-convex in $\mathrm{R}_{\star}^{n}$. Thus $\gamma$ also lies in $\mathrm{B}^{n}(p ; r)$, so $|\gamma| \subset U \cap \mathrm{B}^{n}(p ; r)$.

Now $\gamma$ joins $a \in A$ to $b \in B$ and $|\gamma|$ is connected, so $\gamma$ must meet $\mathrm{P}$. Therefore, $|\gamma| \cap \mathrm{P} \cap \mathrm{B}^{n}(p ; r) \neq \emptyset$. Thus there is a point $x \in|\gamma| \cap \mathrm{P} \cap \mathrm{B}^{n}(p ; r)$, so $x \in U \cap \mathrm{P}$ and hence $x \in V \cap \mathrm{B}^{n}(p ; r)$.

We note that in general a dense open subset of $\mathrm{R}^{n}$ need not have dense intersection with every $(n-1)$-plane; it could even have an empty intersection. That this holds above depends crucially on the quasihyperbolic convexity of $U$ in $\mathrm{R}_{\star}^{n}$. 


\section{Proofs}

Here we prove Theorem $\mathrm{C}$ (which includes Proposition [B), Theorem [A] and Corollary D. We begin by establishing some elementary properties of circle convexity.

3.A. Proof of Theorem $\mathbf{C}$. We note that if $A$ is circle convex in $\hat{\mathrm{R}}^{n}$ and $S$ is a sphere in $\hat{\mathrm{R}}^{n}$ or the closure of an affine subspace of $\mathrm{R}^{n}$, then $A \cap S$ is circle convex in $S$ (provided that $A \cap S \neq \emptyset$ and that $S$ has dimension at least two).

3.1. Lemma. Let $n \geq 2$. Suppose $A$ is circle convex in $\hat{\mathrm{R}}^{n}$. Then $\bar{A}$ is circle convex in $\hat{\mathrm{R}}^{n}$.

Proof. We establish the contrapositive. Assume there is a circle $C$ in $\hat{\mathrm{R}}^{n}$ such that $C \cap \bar{A}$ is not connected. Then there are successive points $a, p, b, q$ along $C$ (in this order) such that $a, b \in \bar{A}$ and $p, q \notin \bar{A}$. Let $T$ be any Möbius transformation that maps $p$ to the origin and $q$ to the point at infinity.

Since $0=T(p) \in \hat{\mathrm{R}}^{n} \backslash \overline{T(A)}$ (which is an open set), there is an open ball $B:=\mathrm{B}(0 ; \varepsilon) \subset \hat{\mathrm{R}}^{n} \backslash \overline{T(A)}$. Since $T(a), T(b) \in \overline{T(A)}$, there are points in $T(A)$ arbitrarily close to $T(a), T(b)$. In particular, we can choose $c, d \in A$ such that the (extended) line $L$ through $T(c)$ and $T(d)$ meets $B$.

Since $B \cap T(A)=\emptyset$ and $\infty \notin T(A)$, we see that $T^{-1}(L) \cap A$ is not connected. Thus $A$ is not circle convex.

3.2. Proof of Proposition $\mathrm{B}$, Let $A$ be a non-empty closed subset of $\hat{\mathrm{R}}^{n}$. If $A$ is the full sphere or a single point or a closed ball, then evidently $A$ is circle convex. We verify the converse. So, suppose that $A$ is circle convex and that $A$ is neither the full sphere nor a single point.

Applying an inversion with respect to any point in $A^{c}$ (if necessary), we may assume that $A$ is bounded; here we use the fact that $A$ is closed to know that each point in $A^{c}$ lies inside an open ball in $A^{c}$. In this setting we must confirm that $A$ is a closed Euclidean ball. Let $B$ be the circumball containing $A$; i.e., $B$ is the smallest closed ball that contains $A$. We show that $A=B$.

Applying a similarity transformation (if necessary), we may assume that $B=\overline{\mathrm{B}}^{n}$. Since $B$ is the circumball about $A$, we know that there exist at least two points in

$$
A \cap \partial B=\partial A \cap \partial B=\partial A \cap \mathrm{S}^{n-1} .
$$

Let $a$ and $b$ be distinct points in $A \cap \partial B$. Since $A$ is circle convex, if $C$ is any circle in $\hat{\mathrm{R}}^{n}$ through $a$ and $b$, then $A \cap C$ is a connected subset of $B$.

Let $p$ be any point in $\mathrm{B}^{n}$. Let $C$ be the circle determined by the three points $a, b, p$. By basic circle geometry, $C \cap \mathrm{S}^{n-1}=\{a, b\}$. This means that $A \cap C$ must be the subarc of $C$ that contains $p$ and has endpoints $a, b$.

Therefore, $p \in A$, and so $\mathrm{B}^{n} \subset A \subset \overline{\mathrm{B}}^{n}$. As $A$ is closed, it follows that $A=\overline{\mathrm{B}}^{n}=$ $B$.

3.3. Proof of Theorem C. We demonstrate that $(2) \Longleftrightarrow(1) \Longleftrightarrow(3)$. That $(1) \Longrightarrow(2)$ follows from the Möbius invariance of circle convexity together with the fact that circle convex sets that lie in $\mathrm{R}^{n}$ are convex. Let $A$ be a non-empty subset of $\hat{\mathrm{R}}^{n}$.

To see that $(2) \Longrightarrow(1)$, suppose that $T(A)$ is convex whenever $T$ is a Möbius transformation with $T(A) \subset \mathrm{R}^{n}$. Let $C$ be a circle in $\hat{\mathrm{R}}^{n}$ with $A \cap C \neq \emptyset$. We show that each pair of points in $A \cap C$ are the endpoints of a subarc of $C$ that lies in $A$. Assume there exists a point $c \in C \backslash A$ and let $a, b \in A \cap C$. 
Let $T$ be any Möbius transformation that maps $c$ to the point at infinity. Then $T(A) \subset \mathrm{R}^{n}$, so $T(A)$ is convex and therefore $[T(a), T(b)] \subset T(A)$. It follows that $T^{-1}([T(a), T(b)]) \subset A$ and this is the desired subarc of $C$.

That $(3) \Longrightarrow(1)$ is straightforward. Clearly, the entire sphere, any once punctured sphere, and any singleton are all circle convex. Suppose $A=B \cup C$, where $B$ is an open Möbius ball, $\partial B \supset C \neq \emptyset$, and either $C$ is connected (if $n=2$ ) or $C$ is circle convex in $\partial B$ (if $n>2$ ). Let $K$ be a circle in $\hat{\mathrm{R}}^{n}$ with $K \cap A \neq \emptyset$.

If $K \cap B \neq \emptyset$, then $\overline{K \cap B}=K \cap \bar{B}$, so $K \cap B \subset K \cap A \subset \overline{K \cap B}$ and since $K \cap B$ is connected, so is $K \cap A$ (by an elementary topology fact). Assume $K \cap B=\emptyset$; then $K \cap \bar{B} \supset K \cap A \neq \emptyset$, so $K \cap \bar{B}$ is either a single point or all of $K$.

If $K \cap \bar{B}=\{c\}$, then $\{c\}=K \cap A$ and is connected. Assume $K=K \cap \bar{B}$; this means $K \subset \partial B$. When $n=2, K=\partial B$ and so $K \cap A=\partial B \cap A=C$ which is connected; if $n>2$, then as $K$ is a circle in $\partial B$ and $C$ is circle convex in $\partial B$, $K \cap A=K \cap C$ is connected.

It remains to corroborate that $(1) \Longrightarrow(3)$; this is the heart of the theorem! Assume that $A$ is circle convex. First, Lemma 3.1 tells us that $\bar{A}$ is also circle convex. Next, Proposition $B$ asserts that either $\bar{A}$ is the entire sphere, or a closed ball, or a single point (in which case so is $A$ ).

Suppose $\bar{A}=\hat{\mathrm{R}}^{n} \neq A$. By inverting with respect to any point in $A^{c}$, we may assume that $A \subset \mathrm{R}^{n}$. Then $A$ is convex and $\bar{A}=\hat{\mathrm{R}}^{n}$, so $A=\mathrm{R}^{n}$. (E.g., if $A$ had a finite boundary point, then there would be a support hyperplane for $A$ and $A$ would lie in some half-space.)

Suppose $\bar{A}=\bar{B}$ for some open ball $B$ in $\hat{\mathrm{R}}^{n}$. We claim that $B \subset A$. For suppose there is some point $p \in B \backslash A$. By taking any circle through $p$ that meets $\partial B$ in two points (and so passes through three points of $A^{c}$ ), we find a point $c \in \partial B \backslash A$. Inverting with respect to the point $c$ permits us to assume that $B$ is an open halfspace in $\mathrm{R}^{n}$ and that $A \subset \mathrm{R}^{n}$. In particular, $A$ is convex. Now $p \in \partial A \cap B$, so there is a support hyperplane for $A$ at $p$, but as $\bar{A}=\bar{B}, A$ must have points on both sides of this supposed support hyperplane.

Finally, assume that $C:=A \cap \partial B \neq \emptyset$. If $n=2$, then $\partial B$ is a circle and so $C$ is connected. If $n>2$, then the remarks in the first paragraph of $\$ 3 . \mathrm{A}$, reveal that $C$ is circle convex in $\partial B$.

\section{B. Proof of Theorem A, We establish the pictured implications.}

The implications $(1) \Longrightarrow(3)$ and $(2) \Longrightarrow(3)$ hold because, $(1)$ on the relevant domains the metrics $\varphi$ and $\mu$ are precisely the appropriate $\tau$. The implications $(4) \Longrightarrow(1)$ and $(4) \Longrightarrow(2)$ are analogs of the results of Jørgensen and<smiles>C=[As](=C)[As]=[Ca]</smiles>
Martin. The main work is in demonstrating that $(3) \Longrightarrow(4)$, and here we utilize Proposition B along with "dimension reduction" arguments.

3.B.1. Proof that (4) $\Longrightarrow(1)$. That Möbius balls are universally Ferrand convex was first established in Jul12. For the reader's convenience we include a proof here.

Let $\Omega \subset \hat{\mathrm{R}}^{n}$ be a quasihyperbolic domain. We prove that closed Möbius balls in $\Omega$ are $\varphi$-convex in $\Omega$; the result for open balls then follows. Suppose B is a Möbius ball with $\overline{\mathrm{B}} \subset \Omega$. We let $x^{*}$ denote the reflection of $x$ across $\partial \mathrm{B}$, and then for any $A \subset \mathrm{R}^{n}, A^{*}:=\left\{a^{*} \mid a \in A\right\}$. 


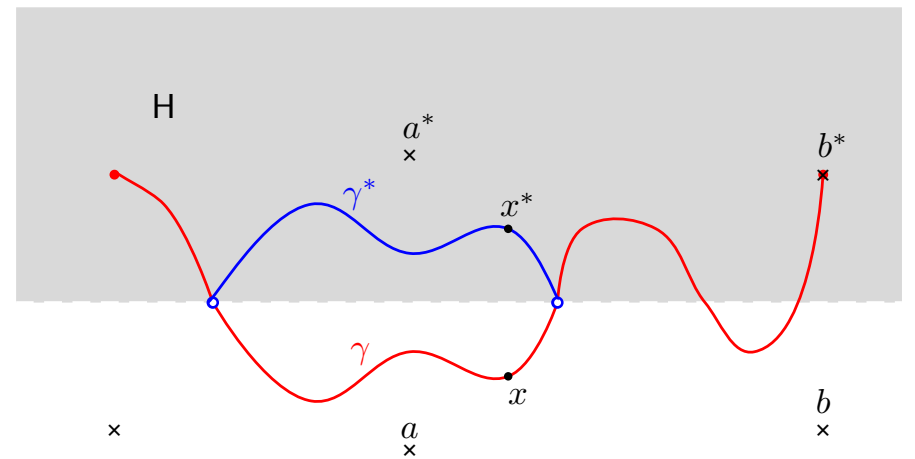

FiguRE 3. $\gamma$ and its reflection $\gamma^{*}$

Suppose we are given a rectifiable path in $\Omega$ that has endpoints in $\bar{B}$ and which leaves B. (See Figure 3.) This path has a subpath $\gamma$ with endpoints in $\partial B$ and such that $\gamma$ only meets $\overline{\mathrm{B}}$ at its endpoints. We demonstrate that $\ell_{\varphi}(\gamma)>\ell_{\varphi}\left(\gamma^{*}\right)$.

By Möbius invariance we may assume that $B$ is the upper half-space $B=\mathrm{H}^{n}$, so $\overline{\mathrm{H}}^{n} \subset \Omega$, and that $|\gamma| \subset \mathrm{R}^{n}$. Then $x \mapsto x^{*}$ is the reflection $R$ across the extended hyperplane $\partial \mathrm{H}^{n}$ (the $\hat{\mathrm{R}}^{n}$-closure of $\mathrm{R}^{n-1} \times\{0\} \subset \mathrm{R}^{n}$ ). Put $\varphi^{*} d s:=R^{\sharp}[\varphi d s]$; so, $\varphi^{*}(x)=\varphi(R(x))\left|R^{\prime}(x)\right|=\varphi\left(x^{*}\right)$.

Since

$$
\ell_{\varphi}(\gamma)=\int_{\gamma} \varphi d s \quad \text { and } \quad \ell_{\varphi}\left(\gamma^{*}\right)=\ell_{\varphi^{*}}(\gamma)=\int_{\gamma} \varphi^{*} d s
$$

it suffices to show that

$$
\int_{\gamma} \varphi d s>\int_{\gamma} \varphi^{*} d s
$$

which in turn follows once we establish that

$$
\forall x \in|\gamma| \backslash \overline{\mathrm{H}}^{n}, \quad \varphi(x)>\varphi^{*}(x)=\varphi\left(x^{*}\right) .
$$

So, let $x \in \Omega \backslash \overline{\mathrm{H}}^{n}$. Pick $a, b \in \partial \Omega$ so that

$$
\varphi\left(x^{*}\right)=\tau_{a b}\left(x^{*}\right)=\frac{|a-b|}{\left|x^{*}-a\right|\left|x^{*}-b\right|} .
$$

Then

$$
\begin{aligned}
\varphi(x) & \geq \tau_{a b}(x)=\tau_{a^{*} b^{*}}\left(x^{*}\right)=\frac{\left|a^{*}-b^{*}\right|}{\left|x^{*}-a^{*}\right|\left|x^{*}-b^{*}\right|} \\
& =\frac{|a-b|}{\left|x^{*}-a\right|\left|x^{*}-b\right|} \frac{\left|x^{*}-a\right|}{\left|x^{*}-a^{*}\right|} \frac{\left|x^{*}-b\right|}{\left|x^{*}-b^{*}\right|} \\
& >\tau_{a b}\left(x^{*}\right)=\varphi\left(x^{*}\right)
\end{aligned}
$$

where the strict inequality above follows because $a, b \notin \overline{\mathrm{H}}^{n}$ whereas $x^{*}, a^{*}, b^{*} \in$ $\mathrm{H}^{n}$. 
3.B.2. Proof that (4) $\Longrightarrow(2)$. As above, let $\Omega \subset \hat{\mathrm{R}}^{n}$ be a quasihyperbolic domain. We prove that closed Möbius balls in $\Omega$ are $\mu$-convex in $\Omega$; the result for open balls then follows. As above, we may assume that the closed upper half-space $\overline{\mathbf{H}}^{n} \subset \Omega$. We demonstrate that

$$
\forall x \in \Omega \backslash \overline{\mathrm{H}}^{n}, \quad \mu(x)>\mu^{*}(x)=\mu\left(x^{*}\right) .
$$

Our argument relies on the technical Lemma 2.12, Let $x \in \Omega \backslash \overline{\mathrm{H}}^{n}$ and let $B:=\mathrm{P}(x)$ be the Kulkarni-Pinkall extremal ball for $x$.

First, suppose $B^{*} \not \subset \Omega$. By Lemma 2.12, $x^{*} \in B$ and $\lambda_{B}(x)>\lambda_{B}\left(x^{*}\right)$; thus

$$
\mu\left(x^{*}\right) \leq \lambda_{B}\left(x^{*}\right)<\lambda_{B}(x)=\mu(x) .
$$

Next, suppose $B^{*} \subset \Omega$. Here we find that

$$
\mu\left(x^{*}\right) \leq \lambda_{B^{*}}\left(x^{*}\right)=\lambda_{B}(x)=\mu(x) .
$$

If $\mu\left(x^{*}\right)=\mu(x)$ were true, then we would have $B^{*}=\mathrm{P}\left(x^{*}\right)$, but then by Lemma 2.12 we would have $x^{*} \in B$ with $\mu(x)=\lambda_{B}(x)>\lambda_{B}\left(x^{*}\right) \geq \mu\left(x^{*}\right)$ (which is a contradiction).

3.B.3. Alternate Proofs that $(4) \Longrightarrow(1),(2)$. The above (analytic) proofs, especially that of $(4) \Longrightarrow(2)$ which relies on Lemma 2.12, do not reveal the underlying geometric reasons that Möbius balls are universally convex for the Ferrand and Kulkarni-Pinkall metrics. Here we present an alternative geometric argument based on Propositions 2.3 and 2.11 and (2.10).

As above, it suffices to demonstrate that for both metric-densities $\rho=\varphi$ and $\rho=\mu$,

$$
\forall x \in \Omega \backslash \overline{\mathrm{H}}^{n}, \quad \rho(x)>\rho^{*}(x)=\rho\left(x^{*}\right)
$$

where $\Omega \subset \hat{\mathrm{R}}^{n}$ is a quasihyperbolic domain that we assume contains the closed upper half-space $\overline{\mathrm{H}}^{n}$. (This inequality implies that closed Möbius balls in $\Omega$ are $\rho$-convex in $\Omega$.)

Fix $x \in \Omega \backslash \overline{\mathrm{H}}^{n}$. Consider $p:=x^{*}$ and $q:=x$ in the setting of Proposition 2.3, now $\mathrm{M}=\partial \mathrm{H}^{n}=\mathrm{P}$. Put $y:=J_{x^{*}}(x)=J_{p}(q)$, then $y^{*}=J_{x}\left(x^{*}\right)=J_{q}(p)$. Let $r:=|y|=$ $\left|y^{*}\right|$. Then $J_{p}(\mathrm{M})=J_{x^{*}}\left(\partial \mathrm{H}^{n}\right)=\mathrm{S}^{n-1}(y ; r)$ and $J_{q}(\mathrm{M})=J_{x}\left(\partial \mathrm{H}^{n}\right)=\mathrm{S}^{n-1}\left(y^{*} ; r\right)$. Also, $R$ is a reflection across $\partial \mathrm{H}^{n}$ and $S$ is a reflection across $\mathrm{S}^{n-1}\left(y^{*} ; r\right)$.

Since $\overline{\mathrm{H}}^{n} \subset \Omega$,

$$
E:=J_{x^{*}}\left(\Omega^{c}\right)=J_{p}\left(\Omega^{c}\right) \subset J_{p}\left(\left(\overline{\mathrm{H}}^{n}\right)^{c}\right)=\left(J_{p}\left(\overline{\mathrm{H}}^{n}\right)\right)^{c}=\mathrm{B}^{n}(y ; r) .
$$

According to (2.4) - which says that $J_{x}=S \circ R \circ J_{x^{*}}$-we have

$$
F:=J_{x}\left(\Omega^{c}\right)=S \circ R \circ J_{x^{*}}\left(\Omega^{c}\right)=S(R(E))
$$

with $R(E) \subset \mathrm{B}^{n}\left(y^{*} ; r\right)$. Appealing to (2.10) and then Proposition 2.11] we deduce that

$$
\varphi(x)=\operatorname{diam} J_{x}\left(\Omega^{c}\right)=\operatorname{diam}(F)>\operatorname{diam} R(E)=\operatorname{diam}(E)=\operatorname{diam} J_{x^{*}}\left(\Omega^{c}\right)=\varphi\left(x^{*}\right)
$$

and similarly

$$
\begin{aligned}
\mu(x) & =\operatorname{cdiam} J_{x}\left(\Omega^{c}\right)=\operatorname{cdiam}(F)>\operatorname{cdiam} R(E) \\
& =\operatorname{cdiam}(E)=\operatorname{cdiam} J_{x^{*}}\left(\Omega^{c}\right)=\mu\left(x^{*}\right) .
\end{aligned}
$$


3.B.4. Proof that (3) $\Longrightarrow(4)$. By hypothesis, $U$ is a non-trivial non-empty open universally $\tau$-convex subset of $\hat{\mathrm{R}}^{n}$. Since there are at least three points in $\hat{\mathrm{R}}^{n} \backslash U$, by Möbius invariance, we may and do assume that these points are $0, \infty, \mathbf{e}_{n}$. That is, we may assume that $U$ is a non-empty open subset of $\mathrm{R}_{\star}^{n}$ with $\mathbf{e}_{n} \notin U$ and $U$ universally $\tau$-convex.

First we show that $\bar{U} \neq \hat{\mathrm{R}}^{n}$. Next we show that $\bar{U}$ is circle convex in $\hat{\mathrm{R}}^{n}$. Then we deduce that $U$ is an open Möbius ball.

We claim that $U$ is not dense in $\hat{\mathrm{R}}^{n}$. This is not difficult to check when $n=2$ (see below). We use a "dimension reduction" argument to reduce our dimension to this case. Let $\mathrm{P}$ be any $(n-1)$-plane in $\mathrm{R}^{n}$ that contains $\mathbf{e}_{n}$. According to Lemma 2.13, $V:=U \cap \mathrm{P}$ is a non-trivial non-empty open $k$-convex subset of $\mathrm{P}_{\star}:=\mathrm{P} \backslash\{0\}$, and if $\bar{U}=\hat{\mathrm{R}}^{n}$, then $\bar{V}=\hat{\mathrm{P}}$. We can repeat this process until we produce a non-empty open $V \subset \mathrm{R}_{\star}^{2}$ with $\mathbf{e}_{2}=(0,1) \notin V$ and $V k$-convex in $\mathrm{R}_{\star}^{2}$. We claim that for such a $V, \bar{V} \neq \hat{\mathrm{R}}^{2}\left(\right.$ so $\left.\bar{U} \neq \hat{\mathrm{R}}^{n}\right)$.

Since every circular arc centered at the origin (with angular length at most $\pi$ ) is a $k$-geodesic in $\mathrm{R}_{\star}^{2}$ and $\mathbf{e}_{2} \notin V$, it follows that there is a semi-circle $C$ with $\mathbf{e}_{2} \in C \subset \mathrm{S}^{1} \backslash V$. If $V$ were dense in $\hat{\mathrm{R}}^{2}$, then we could find points $a, b \in V$ with $|a|<1<|b|$ and $a, b$ close to the midpoint of $C$, but then the $k_{\star}$-geodesic joining $a$ and $b$ would cross $C$ contradicting $C \cap V=\emptyset$. Thus $V$ is not dense in $\hat{\mathrm{R}}^{2}$.

It now follows that our original $U$ is not dense in $\hat{\mathrm{R}}^{n}$.

Next we verify that $A:=\bar{U}$ is circle convex in $\hat{\mathrm{R}}^{n}$. Since $\bar{U} \neq \hat{\mathrm{R}}^{n}$, Möbius invariance permits us to assume that $A$ is a bounded subset of $\mathrm{R}^{n}$. Let $C$ be any circle in $\hat{\mathrm{R}}^{n}$. We may assume that $C \cap A \neq \emptyset \neq C \backslash A$. Using Möbius invariance again, we may invert with respect to any point in $C \backslash A$ and thus assume that

$$
C \text { is an extended line in } \hat{\mathrm{R}}^{n} \text { and } A \text { is a bounded subset of } \mathrm{R}^{n} \text {. }
$$

We explain why $A \cap C$ is connected.

Since $A$ is bounded, it lies inside some Euclidean ball $B$. Fix $p \in C \backslash B$. Note that for any $a, b \in A \cap C,[a, b] \subset B$ and so $p \notin[a, b]$.

Now $U \subset \mathrm{R}_{p}^{n}:=\mathrm{R}^{n} \backslash\{p\}, U$ is universally $\tau$-convex, and $\tau_{p \infty}(x)=1 /|x-p|$, so $U$ is $k_{p}$-convex where $k_{p}:=k_{\mathrm{R}_{p}^{n}}$ (i.e., $U$ is $k$-convex in $\mathrm{R}_{p}^{n}$ ). Since each component of $C \backslash\{p\}$ is a $k_{p}$-geodesic (being an infinite ray from $p$ ), it follows that $U \cap C$ is connected.

To see that $A \cap C$ is connected, we show that $a, b \in A \cap C$ implies that $[a, b] \subset$ $A \cap C$. So, let $a, b \in A \cap C$. Recall that for such $a, b$ we know that $p \notin[a, b]$, so $[a, b]$ is the unique $k_{p}$-geodesic with endpoints $a, b$. Pick sequences $\left(a_{i}\right),\left(b_{i}\right)$ in $U$ with $a_{i} \rightarrow a$ and $b_{i} \rightarrow b$. Let $\gamma_{i}$ be $k_{p}$-geodesics with endpoints $a_{i}, b_{i}$. Then each $\gamma_{i}$ lies in $U$. According to [BH99, Lemma 3.12, p. 37], $\left(\gamma_{i}\right)$ converges uniformly to the $k_{p}$-geodesic $\gamma$ joining $a, b$. Thus each point of $|\gamma|=[a, b]$ is a limit of some sequence $\left(\gamma_{i}(t)\right)$ of points in $U$. Therefore, $[a, b] \subset A \cap C$.

Since $A=\bar{U}$ is circle convex in $\hat{\mathrm{R}}^{n}$, Proposition $\mathrm{B}$ tells us that $A$ is a closed Möbius ball. By Möbius invariance, we may assume that $\bar{U}=\overline{\mathrm{B}}^{n}$, so $U \subset \mathrm{B}^{n}$. Finally, we verify that $\mathrm{B}^{n} \subset U$; thus $U=\mathrm{B}^{n}$. Using Möbius invariance one more time, it suffices to demonstrate that the origin lies in $U$.

Suppose that $0 \notin U$. By repeated applications of Lemma 2.13 (here we use part (ii)), we can reduce the dimension to the case where we have a non-empty open $U \subset \mathrm{R}_{\star}^{2}$ with $U k_{\star}$-convex (i.e., $k$-convex in $\mathrm{R}_{\star}^{2}$ ) and $\bar{U}=\overline{\mathrm{B}}^{2}$. Now, for any $\zeta \in \partial \mathrm{B}^{2},(0, \zeta)$ is a $k_{\star}$-geodesic, so $(0, \zeta) \cap U$ is connected (i.e., an interval). 
However, from Lemma 2.13 (applied to $U$ and the "hyperplane" $\operatorname{Span}\{\zeta\} \subset \mathrm{R}^{2}$ ) we see that $(0, \zeta) \cap U$ is dense in $(0, \zeta)$. Since $(0, \zeta) \cap U$ is an interval, it follows that $(0, \zeta) \cap U=(0, \zeta)$, and this holds for each $\zeta \in \partial \mathrm{B}^{2}$.

This means that $U=\mathrm{B}_{\star}^{2}:=\mathrm{B}^{2} \backslash\{0\}$. However, $\mathrm{B}_{\star}^{2} \subset \mathrm{R}_{1}^{2}:=\mathrm{R}^{2} \backslash\{1\}$ and evidently $\mathrm{B}_{\star}^{2}$ is not $k$-convex in $\mathrm{R}_{1}^{2}$. This contradiction means that the origin lies in $U$.

3.C. Proof of Corollary D. Let $\mathcal{M}=\left\{\rho_{\Omega} d s\right\}_{\Omega \in \mathcal{O}}$ be a Möbius invariant class of conformal metrics with $\mathrm{R}^{n} \backslash\{0\} \in \mathcal{O}$. Since $\mathcal{O}$ is Möbius invariant, it follows that $\mathcal{O}$ contains the collection $\mathcal{S}_{\star}$ of all twice punctured spheres $\hat{\mathrm{R}}_{a b}^{n}$. In particular, $\left\{\rho_{\Omega} d s\right\}_{\Omega \in \mathcal{S}_{\star}}$ is a Möbius invariant class of conformal metrics.

According to Corollary 2.6. there is a constant $C$ such that for each $\Omega=\hat{\mathrm{R}}_{a b}^{n} \in \mathcal{S}_{\star}$, $\rho_{\Omega} d s=C \tau_{a b} d s$. Therefore, any $U$ that is universally convex with respect to $\mathcal{M}$ is also universally convex with respect to $\mathcal{T}$, and hence, when non-trivial, is an open ball in $\hat{\mathrm{R}}^{n}$.

\section{EXAMPLES}

Here we exhibit a number of large Möbius invariant classes of complete continuous conformal metrics $\rho d s$ all of which satisfy the assertions stated in Example E. Our examples are all based on the following construction of a Möbius invariant metric on the punctured unit ball $B_{\star}^{n}:=B^{n} \backslash\{0\}$.

4.1. Lemma. There exists a complete Möbius invariant conformal metric $\rho_{\star} d s$ on the punctured ball $\mathrm{B}_{\star}^{n}$ with the property that for each $\varepsilon>0$ there exists an $r \in(0, \varepsilon]$ and a Euclidean ball $\mathrm{B}^{n}(a ; r) \subset \mathrm{B}_{\star}^{n}$ which is not $\rho$-convex.

Proof. Since the only self-Möbius transformations of $\mathrm{B}_{\star}^{n}$ are orthogonal transformations (see [Bea83, Theorem 3.4.1, p. 38]), it suffices to produce a radial metric with the desired property. Define

$$
\rho_{\star}(x):= \begin{cases}g(|x|) & \text { when } 0<|x| \leq \frac{1}{2}, \\ \frac{5}{1-|x|} & \text { when } \frac{1}{2} \leq|x|<1\end{cases}
$$

where $(0,1 / 2] \stackrel{g}{\rightarrow}(0, \infty)$ is the piecewise linear function whose graph is pictured in Figure 4. (Instead of the quasihyperbolic metric $(1-|x|)^{-1}$ we could, e.g., use the hyperbolic metric on $\mathrm{B}^{n}$ or the hyperbolic metric on $\mathrm{B}_{\star}^{n}$.)

Since there is no harm in doing so, to simplify notation we work in the plane $\mathrm{R}^{2}$ which we identify with the complex number field C. Especially, we use complex variables notation. For each natural number $n \in \mathrm{N}$, let

$$
t_{n}:=\frac{1}{2^{n}} \quad \text { and } \quad r_{n}:=\frac{t_{n}-t_{n+1}}{2}=\frac{t_{n+1}}{2}=\frac{t_{n}}{4} .
$$

The piecewise linear function $g$ is defined by setting $g$ identically equal to $R_{n}:=10^{n}$ on the interval $\left[t_{n+1}+r_{n+1}^{4}, t_{n}\right]$.

Next, put

$$
a_{n}:=t_{n}-r_{n}=3 r_{n} \quad \text { and } \quad D_{n}:=\mathrm{B}^{2}\left(a_{n} ; r_{n}\right)
$$

and select $z_{n} \in \partial D_{n}$ (as pictured in Figure 5) so that

$$
\Im \mathfrak{m}\left(z_{n}\right)=r_{n}^{2} \text {. }
$$




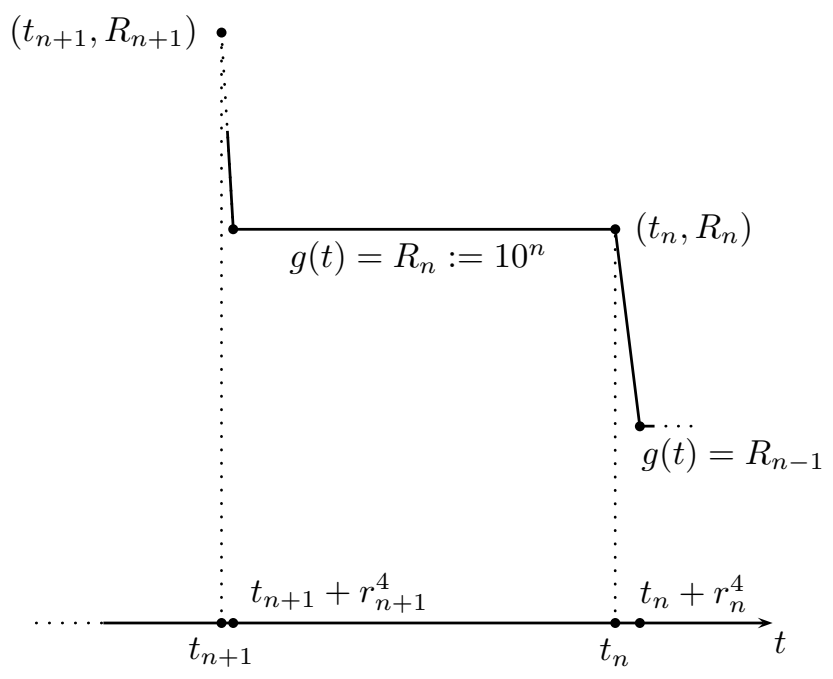

FiguRE 4. Graph of $g$

Then $\theta_{n}:=\operatorname{Arg}\left(z_{n}\right) \in(0, \pi / 2)$ satisfies

$$
\left|z_{n}\right| \sin \theta_{n}=r_{n}^{2}
$$

and since $\left|z_{n}\right| \geq a_{n}=3 r_{n}$, we find that

$$
r_{n} \geq 3 \sin \theta_{n} \geq \theta_{n} .
$$

We demonstrate that for all $n \geq 2$, every $\rho$-geodesic between $z_{n}$ and $\bar{z}_{n}$ leaves the disk $\bar{D}_{n}$. Therefore, $D_{n}$ is not $\rho$-convex in $\mathrm{B}_{\star}^{2}$.

First we note that as $\rho \geq R_{n}$ in $\bar{D}_{n}$, any path in $\bar{D}_{n}$ that joins $z_{n}$ and $\bar{z}_{n}$ has $\rho$-length at least $R_{n}\left|z_{n}-\bar{z}_{n}\right|=2 R_{n}\left|z_{n}\right| \sin \theta_{n}$. We exhibit a path $\gamma_{n}$ in $\mathrm{B}_{\star}^{2} \backslash D_{n}$ that joins the points $z_{n}, \bar{z}_{n}$ and has $\rho$-length strictly smaller than $R_{n}\left|z_{n}-\bar{z}_{n}\right|$.

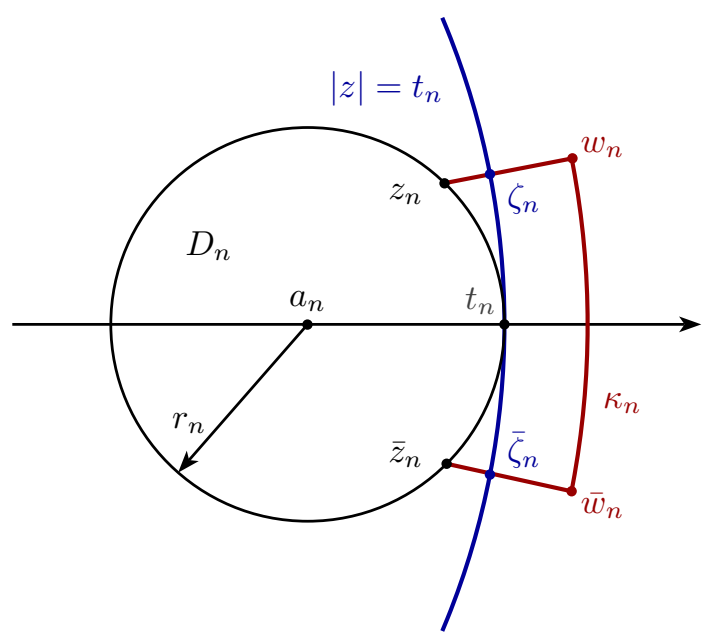

Figure 5. The path $\gamma_{n}:=\left[z_{n}, w_{n}\right] \cup \kappa_{n} \cup\left[\bar{w}_{n}, \bar{z}_{n}\right]$ 
To this end (see Figure 5) set

$$
\zeta_{n}:=t_{n} e^{i \theta_{n}}, w_{n}:=\left(t_{n}+r_{n}^{4}\right) e^{i \theta_{n}}
$$

and let $\kappa_{n}$ be the shorter subarc of the circle $|z|=t_{n}+r_{n}^{4}$ with endpoints $w_{n}, \bar{w}_{n}$. Then

$$
\gamma_{n}:=\left[z_{n}, w_{n}\right] \cup \kappa_{n} \cup\left[\bar{w}_{n}, \bar{z}_{n}\right]
$$

is a rectifiable path in $\mathrm{B}_{\star}^{2} \backslash D_{n}$ that joins $z_{n}$ and $\bar{z}_{n}$. To show that

$$
\ell_{\rho}\left(\gamma_{n}\right)<R_{n}\left|z_{n}-\bar{z}_{n}\right|=2 R_{n}\left|z_{n}\right| \sin \theta_{n}=2 R_{n} r_{n}^{2}
$$

we estimate $\ell_{\rho}\left(\left[z_{n}, \zeta_{n}\right]\right), \ell_{\rho}\left(\left[\zeta_{n}, w_{n}\right]\right)$, and $\ell_{\rho}\left(\kappa_{n}\right)$.

Evidently,

$$
\begin{gathered}
\ell_{\rho}\left(\kappa_{n}\right)=2 \theta_{n} R_{n-1}\left(t_{n}+r_{n}^{4}\right), \\
\ell_{\rho}\left(\left[\zeta_{n}, w_{n}\right]\right) \leq R_{n}\left|\zeta_{n}-w_{n}\right|=R_{n} r_{n}^{4}, \\
\ell_{\rho}\left(\left[z_{n}, \zeta_{n}\right]\right)=R_{n}\left|z_{n}-\zeta_{n}\right|,
\end{gathered}
$$

and so (recalling that $R_{n}=10 R_{n-1}$ ) we see that

$$
\ell_{\rho}\left(\gamma_{n}\right) \leq 2 R_{n}\left[\frac{\theta_{n}}{10}\left(t_{n}+r_{n}^{4}\right)+r_{n}^{4}+\left|z_{n}-\zeta_{n}\right|\right] .
$$

It is not difficult to check that $\left|z_{n}-\zeta_{n}\right|<r_{n}^{3}$. Using this in conjunction with $\theta_{n} \leq r_{n}$ and $t_{n}=4 r_{n}$ we see that

$$
\ell_{\rho}\left(\gamma_{n}\right) \leq 2 R_{n}\left[\frac{4}{10} r_{n}^{2}+\frac{1}{10} r_{n}^{5}+r_{n}^{4}+r_{n}^{3}\right]<2 R_{n} r_{n}^{2}
$$

here the above right-hand strict inequality holds because

$$
\forall n \geq 1, \quad \frac{1}{10} r_{n}^{3}+r_{n}^{2}+r_{n}<3 r_{n}=\frac{3}{2^{n+2}}<\frac{3}{5} .
$$

Obviously, we can use Möbius transformations to transport $\rho_{\star} d s$ to any punctured Möbius ball. For example, by reflecting across $\partial \mathrm{B}_{\star}^{n}=\mathrm{S}^{n-1}$ we obtain a Möbius invariant metric $|x|^{-2} \rho_{\star}\left(x^{\star}\right)|d x|$ on $\mathrm{R}^{n} \backslash \overline{\mathrm{B}}^{n}$ (see (2.2) ), and there are arbitrarily large Euclidean balls in $\mathrm{R}^{n} \backslash \overline{\mathrm{B}}^{n}$ that are not convex with respect to this metric. See also Examples 4.2, 4.3, 4.4,

We can mimic the above construction to create similar metrics, e.g., on spherical rings (such as $\left\{x \in \mathrm{R}^{n}|1<| x \mid<2\right\}$ ) and on infinite slabs (such as $\left\{\left(x_{1}, \ldots, x_{n}\right) \in\right.$ $\left.\mathrm{R}^{n}|| x_{n} \mid<1\right\}$ ), as well as on Möbius images of these. Here, though we must take into account that reflection across the "center sphere" (or "center hyperplane"), is a Möbius self-map. This "works" because we know the automorphism groups for these types of regions.

It is tempting to try to use the above idea to construct such a metric on the twice punctured sphere $\mathrm{R}_{\star}^{n}$, but as the alert reader recognizes, this will fail because dilations are also self-maps of $\mathrm{R}_{\star}^{n}$. Indeed, according to Lemma 2.5, in conjunction with Martin's theorem, Euclidean balls are always $\rho$-convex for any Möbius invariant metric $\rho d s$ on $\mathrm{R}_{\star}^{n}$.

Now we describe several classes of complete Möbius invariant conformal metrics, all of which satisfy the assertions stated in Example E. 
Recall that $\mathcal{B}$ is the collection of all Möbius balls in $\hat{\mathrm{R}}^{n}$. For each $p \in B \in \mathcal{B}$, put $B_{p}:=B \backslash\{p\}$ and set

$$
\mathcal{B}_{\star}:=\left\{B_{p} \mid p \in B \in \mathcal{B}\right\} .
$$

For each $B_{p} \in \mathcal{B}_{\star}$, select a Möbius transformation $T$ that maps $B_{p} \cup\{p\}$ to $\mathrm{B}^{n}$ with $T(p)=0$. Then $\rho_{B_{p}} d s:=T^{*}\left[\rho_{\star} d s\right]$ is a continuous complete Möbius invariant metric on $B_{p}$ and we obtain the following.

4.2. Exercise. There is a Möbius invariant class $\mathcal{M}_{\star}:=\left\{\rho_{B_{p}} d s\right\}_{B_{p} \in \mathcal{B}_{\star}}$ of complete conformal metrics such that each $B_{p}$ contains infinitely many Möbius balls that are not $\rho$-convex.

Now we turn to examples involving punctured spheres. We restrict our attention to $\hat{\mathrm{R}}^{2}$ which we identify with $\hat{\mathrm{C}}$. Suppose $\Omega:=\hat{\mathrm{C}} \backslash F$ where $F \subset \hat{\mathrm{C}}$ is a finite set with $\operatorname{card}(F) \geq 4$. It is known (see BM08) that "most" such domains have a trivial automorphism group: the only Möbius self-map of "most" such regions is the identity. It is easy to produce a complete Möbius invariant conformal metric $\rho d s$ on such an $\Omega$ with the property that $\Omega$ contains many Möbius balls that are not $\rho$-convex. Indeed, we simply pick disjoint disks centered at the points of $F$, transport $\rho_{\star} d s$ (the metric from Lemma 4.1) to each of these disks, and then paste all these metrics together by using the underlying hyperbolic metric $\lambda d s=\lambda_{\Omega} d s$; see below for ideas of how to do this pasting.

The triply punctured spheres $\hat{\mathrm{C}}_{a b c}:=\hat{\mathrm{C}} \backslash\{a, b, c\}$ form an important class of domains that possess non-trivial automorphism groups, so the above argument does not work. For example, the conformal automorphism group for $\hat{C}_{1 \omega \bar{\omega}}\left(\omega:=e^{2 \pi i / 3}\right)$ is precisely $\left\{I, J, R, R^{2}, R \circ J, J \circ R\right\}$ where $I(z):=z, J(z):=1 / z$, and $R(z):=\omega z$. Nonetheless, we have the following.

4.3. Exercise. There are complete Möbius invariant conformal metrics $\rho_{a b c} d s$, defined on the triply punctured spheres $\hat{\mathrm{C}}_{a b c}$, such that each $\hat{\mathrm{C}}_{a b c}$ contains infinitely many Möbius balls that are not $\rho_{a b c}$-convex, and $\left\{\rho_{a b c} d s \mid a, b, c\right.$ distinct points in $\left.\hat{\mathrm{C}}\right\}$ is Möbius invariant.

Our construction for Example 4.3 generalizes to give Example 4.4 as stated below. Here $n \geq 3$ is a fixed integer, $\omega=\omega_{n}:=\exp (2 \pi i / n)$,

$$
\Omega_{n}:=\hat{\mathrm{C}} \backslash\left\{1, \omega, \omega^{2}, \ldots, \omega^{n-1}\right\} \quad \text { and } \mathcal{S}_{n}:=\left\{T\left(\Omega_{n}\right) \mid T \in \operatorname{Möb}(\hat{\mathrm{C}})\right\} .
$$

4.4. Exercise. There are complete Möbius invariant conformal metrics $\rho_{\Omega} d s$, defined on each $\Omega \in \mathcal{S}_{n}$, such that each $\Omega$ contains infinitely many Möbius balls that are not $\rho_{\Omega}$-convex, and $\left\{\rho_{\Omega} d s \mid \Omega \in \mathcal{S}_{n}\right\}$ is Möbius invariant.

Proof. Evidently, it suffices to construct an appropriate metric on $\Omega=\Omega_{n}$ (which can then be transported to each Möbius image of $\Omega$ ). We note that the conformal automorphism group for $\Omega$ is generated by $J$ and $R$ where $J(z):=1 / z$ and $R(z):=$ $\omega z$.

Let $D:=\mathrm{B}^{2}(c ; r)$ where $c>1$ and $r>0$ are chosen so that the circle $\partial D$ meets $\mathrm{S}^{1}=\partial \mathrm{B}^{2}$ orthogonally and so that $\bar{D} \cap \omega \bar{D}=\emptyset$. (The first of these conditions holds provided $c^{2}=1+r^{2}$, and the second condition holds as soon as $r<\tan (\pi / n)$.) See Figure 6.

Let $T$ be the Möbius transformation that satisfies

$$
T(D)=\mathrm{B}^{2}, T(1)=0, T\left(\mathrm{~S}^{1} \cap D\right)=(-1,1), T\left(\mathrm{~B}^{2} \cap D\right)=\mathrm{H}^{2} \cap \mathrm{B}^{2} .
$$




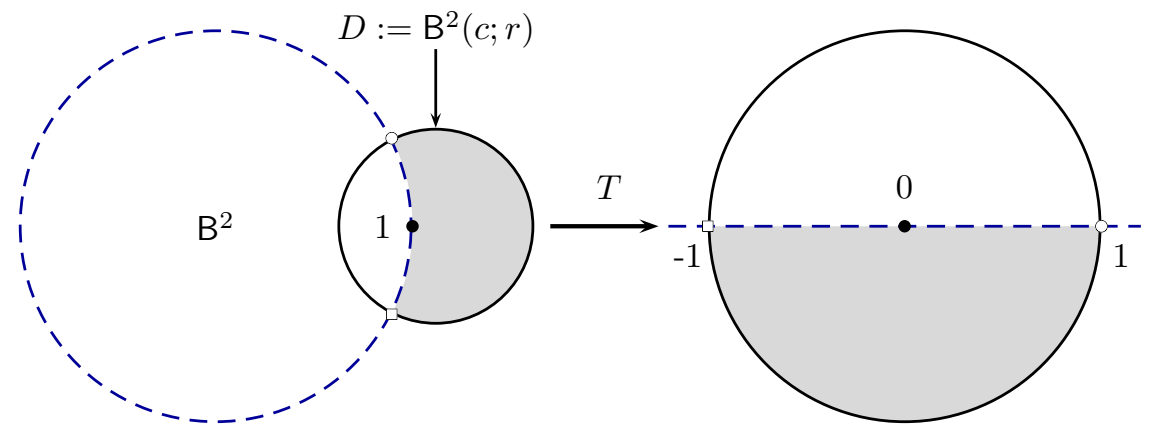

Figure 6 . The ball $D=\mathrm{B}^{2}(c ; r)$ and Möbius map $T$

Then $T(-1)=\infty$, so $T(z)=i \kappa(z-1) /(z+1)$ for some $\kappa \in(-\infty,-1)$ and one can calculate $\kappa$. Also, $T\left(\mathrm{~S}^{1}\right)=\hat{\mathrm{R}}, T(\hat{\mathrm{R}})=i \hat{\mathrm{R}}$, and $T(1 / z)=-T(z)$, so $T^{\prime}(1 / z)=z^{2} T^{\prime}(z)$.

For each $k \in\{1, \ldots, n\}$, set $D_{k}:=\omega^{k} D$ (so $D_{1}, D_{2}, \ldots, D_{n}=D$ are pairwise disjoint disks centered at the points $\omega^{k} c$ and each of radius $r$ ) and put $T_{k}:=T \circ R^{-k}$ (so $T_{n}=T$ and $T_{k}$ maps $D_{k}$ onto $\mathrm{B}^{2}$ with $T_{k}\left(\omega^{k}\right)=0$ ). See Figure 7 We transport $\rho_{\star} d s$ (constructed in Lemma 4.1) to $D_{k}^{\prime}:=D_{k} \backslash\left\{\omega^{k}\right\}$ by using $T_{k}$. Put $\rho_{k} d s:=T_{k}^{\sharp}\left[\rho_{\star} d s\right]$; that is,

$$
\text { for } z \in D_{k}^{\prime} \text {, define } \rho_{k}(z):=\rho_{\star}\left(T_{k}(z)\right)\left|T_{k}^{\prime}(z)\right| .
$$

For future reference, we remind the reader that $\rho_{\star}$ is constant on each circle $|z|=$ $r \in(0,1)$.

We note that each metric $\rho_{k} d s$ is $J$-invariant, and evidently, $R^{\sharp}\left[\rho_{k} d s\right]=\rho_{k-1} d s$.

We use the underlying hyperbolic metric $\lambda d s=\lambda_{\Omega} d s$ to "paste together" the metrics $\rho_{k} d s$ and define a metric $\rho d s$ on all of $\Omega$. To this end, for each $k \in$ $\{1, \ldots, n\}$, set

$$
C_{k}:=T_{k}^{-1}(\{|z|=1 / 2\}) \quad \text { and } \quad A_{k}:=T_{k}^{-1}(\{1 / 2<|z|<1\}) ;
$$

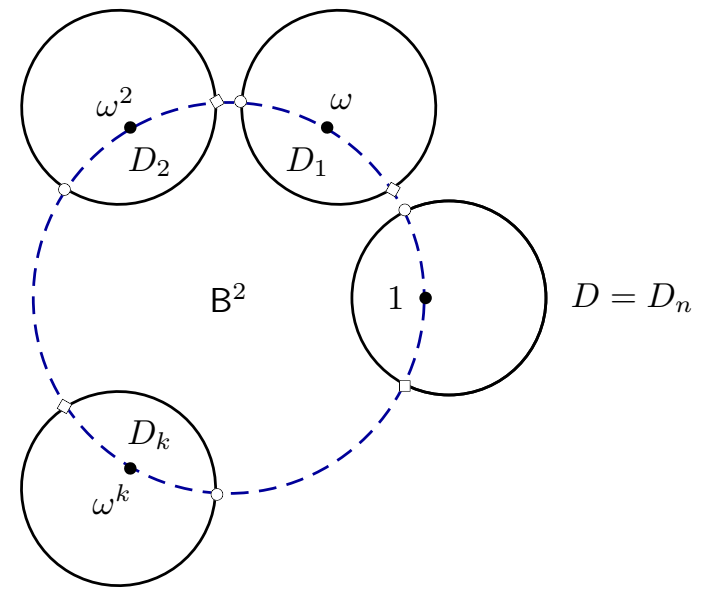

Figure 7. The disjoint balls $D_{1}, \ldots, D_{n}$ 


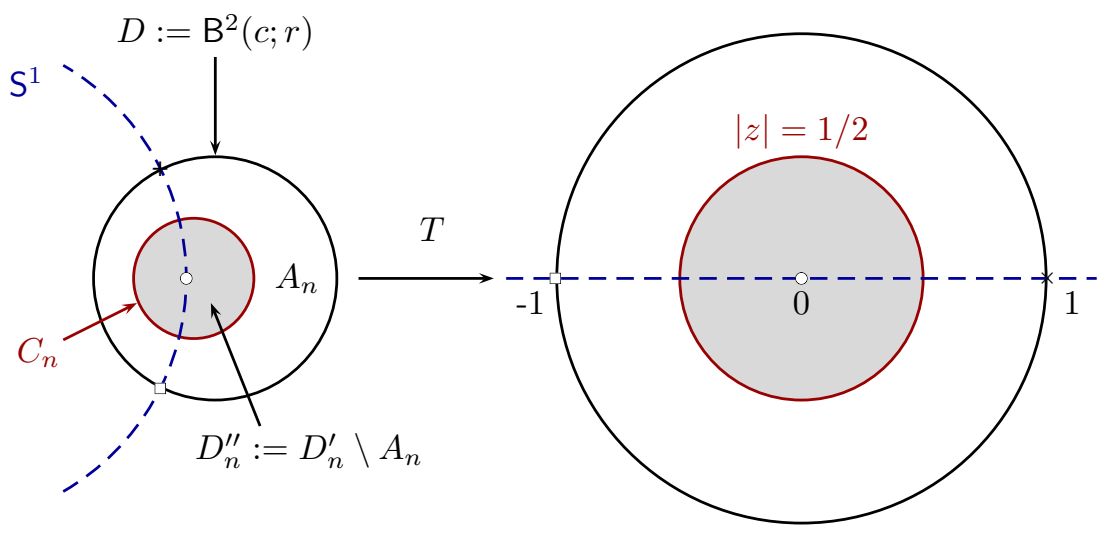

Figure 8 . The annulus $A_{n}$, circle $C_{n}$, punctured disk $D_{n}^{\prime \prime}$

thus each $A_{k} \subset \Omega$ is a Möbius annulus with $\partial A_{k}=C_{k} \cup \partial D_{k}$. See Figure 8, Note that as $K:=\left.\rho_{\star}\right|_{\{|z|=1 / 2\}}$ is a constant,

$$
\forall z \in C_{k}, \quad \rho_{k}(z)=K\left|T_{k}^{\prime}(z)\right|=K\left|T^{\prime}(z)\right| .
$$

Set

$$
m:=\inf _{z \in D_{n}^{\prime}} \lambda(z)=\inf \left\{\lambda(z) \mid z \in D_{1}^{\prime} \cup \cdots \cup D_{n}^{\prime}\right\}
$$

and

$$
M:=\max _{z \in C_{n}}\left|T^{\prime}(z)\right|=\max _{z \in C_{k}}\left|T_{k}^{\prime}(z)\right| ; \quad \text { so } \quad K M=\max _{z \in C_{k}}\left|\rho_{k}(z)\right| .
$$

Note that as $z \rightarrow 1, \lambda(z) \rightarrow+\infty$, so $m>0$. Fix a constant $C>K M / m$.

Now, in $D_{k}^{\prime}=D_{k} \backslash\left\{\omega^{k}\right\}$,

$$
C \lambda \geq C m>K M, \quad \text { and on } C_{k}, \quad K M \geq \rho_{k} .
$$

It follows that we can define $\sigma_{k}$ in $D_{k}^{\prime}$ by

$$
\sigma_{k}:= \begin{cases}\rho_{k} & \text { in } D_{k}^{\prime \prime}:=D_{k}^{\prime} \backslash A_{k}, \\ \min \left\{\rho_{k}, C \lambda\right\} & \text { in } A_{k},\end{cases}
$$

and $\sigma_{k}$ is continuous (and clearly positive) in $D_{k}^{\prime}$.

As points $z \in D_{k}$ approach $\partial D_{k}, T_{k}(z)$ approaches $\mathrm{S}^{1}=\partial \mathrm{B}^{2}$, so $\rho_{\star}\left(T_{k}(z)\right) \rightarrow$ $+\infty$. As $\left|T_{k}^{\prime}(z)\right|$ is bounded away from 0 (for $z \in \bar{D}_{k}$ ), there are neighborhoods $U_{k}$ of $\partial D_{k}$ in $A_{k}$ such that

$$
\forall z \in U_{k}, \quad \sigma_{k}(z)=C \lambda(z) .
$$

In particular, this means that we can define a continuous positive $\rho$ on $\Omega \backslash\{\infty\}$ by

$$
\rho:=\left\{\begin{array}{ll}
\sigma_{k} & \text { in } D_{k}^{\prime}, \\
C \lambda & \text { in } \Omega \backslash\left(\{\infty\} \cup \bigcup_{1}^{n} D_{k}\right)
\end{array}= \begin{cases}\rho_{k} & \text { in } D_{k}^{\prime \prime}, \\
\min \left\{\rho_{k}, C \lambda\right\} & \text { in } A_{k}, \\
C \lambda & \text { in } \Omega \backslash\left(\{\infty\} \cup \bigcup_{1}^{n} D_{k}\right) .\end{cases}\right.
$$

Finally, $\rho$ is invariant with respect to both $J$ and $R$; i.e., $(\rho \circ J)\left|J^{\prime}\right|=\rho=$ $(\rho \circ R)\left|R^{\prime}\right|$. In particular, $\rho$ induces a continuous complete Möbius invariant metric 
$\rho d s$ defined on all of $\Omega$, and near each point of $\partial \Omega$ (i.e., near each of the punctures $\left.\omega^{k}\right)$ there are arbitrarily small Euclidean disks in $\Omega$ which fail to be $\rho$-convex.

\section{ACKNOWLEDGEMENT}

The author thanks David Minda for interesting discussions about this article, and especially for three decades worth of answers to many questions.

\section{REFERENCES}

[Bea83] A. F. Beardon, The geometry of discrete groups, Graduate Texts in Mathematics, vol. 91, Springer-Verlag, New York, 1983. MR698777 (85d:22026)

[BM08] A. F. Beardon and D. Minda, Conformal automorphisms of finitely connected regions, Transcendental dynamics and complex analysis, London Math. Soc. Lecture Note Ser., vol. 348, Cambridge Univ. Press, Cambridge, 2008, pp. 37-73, DOI 10.1017/CBO9780511735233.004. MR2458798 (2010c:30061)

[Ber87] M. Berger, Geometry I, Springer, Berlin, 1987.

[BH99] M. R. Bridson and A. Haefliger, Metric spaces of non-positive curvature, Grundlehren der Mathematischen Wissenschaften [Fundamental Principles of Mathematical Sciences], vol. 319, Springer-Verlag, Berlin, 1999. MR.1744486 (2000k:53038)

[Fer88] J. Ferrand, A characterization of quasiconformal mappings by the behaviour of a function of three points, Complex analysis, Joensuu 1987, Lecture Notes in Math., vol. 1351, Springer, Berlin, 1988, pp. 110-123, DOI 10.1007/BFb0081247. MR982077|(89m:30040)

[Fli83] B. B. Flinn, Hyperbolic convexity and level sets of analytic functions, Indiana Univ. Math. J. 32 (1983), no. 6, 831-841, DOI 10.1512/iumj.1983.32.32056. MR721566 (85b:30010)

[HIM08] D. A. Herron, Z. Ibragimov, and D. Minda, Geodesics and curvature of Möbius invariant metrics, Rocky Mountain J. Math. 38 (2008), no. 3, 891-921, DOI 10.1216/RMJ2008-38-3-891. MR2426525 (2009h:30079)

[HJ13] D. A. Herron and P. K. Julian, Ferrand's Möbius invariant metric, J. Anal. 21 (2013), 101-121. MR3408021

[HMM03] D. A. Herron, W. Ma, and D. Minda, A Möbius invariant metric for regions on the Riemann sphere, Future trends in geometric function theory, Report. University of Jyväskylä Department of Mathematics and Statistics, vol. 92, University of Jyväskylä, Jyväskylä, 2003. MR2060255 (2004k:30001)

[HMM05] D. A. Herron, W. Ma, and D. Minda, Estimates for conformal metric ratios, Comput. Methods Funct. Theory 5 (2005), no. 2, 323-345, DOI 10.1007/BF03321101. MR.2205417(2006j:30082)

[HMM08] D. A. Herron, W. Ma, and D. Minda, Möbius invariant metrics bilipschitz equivalent to the hyperbolic metric, Conform. Geom. Dyn. 12 (2008), 67-96, DOI 10.1090/S10884173-08-00178-1. MR2410919 (2009b:30090)

[Jør56] V. Jørgensen, On an inequality for the hyperbolic measure and its applications in the theory of functions, Math. Scand. 4 (1956), 113-124. MR0084584(18,885a)

[Jul12] P. K. Julian, Geometric Properties of the Ferrand Metric, Ph.D. thesis, University of Cincinnati, 2012.

[KP94] R. S. Kulkarni and U. Pinkall, A canonical metric for Möbius structures and its applications, Math. Z. 216 (1994), no. 1, 89-129, DOI 10.1007/BF02572311. MR.1273468 (95b:53017)

[Mar85] G. J. Martin, Quasiconformal and bi-Lipschitz homeomorphisms, uniform domains and the quasihyperbolic metric, Trans. Amer. Math. Soc. 292 (1985), no. 1, 169-191, DOI 10.2307/2000176. MR805959 (87a:30037)

Department of Mathematical Sciences, French Hall West, PO Box 210025, Cincinnati Оніо 45221-0025

E-mail address: David.Herron@UC.edu 